\title{
Rare decays of $\Lambda_{b} \rightarrow \Lambda+\gamma$ and $\Lambda_{b} \rightarrow \Lambda+l^{+} l^{-}$in the light-cone sum rules
}

\author{
Yu-Ming Wang ${ }^{1}$, Ying $\mathrm{Li}^{2}$, and Cai-Dian Lü ${ }^{1}$ \\ ${ }^{1}$ Institute of High Energy Physics, P.O. Box 918(4), Beijing 100049, China and \\ ${ }^{2}$ Physics Department, Yantai University, Yantai, 264005, China
}

\begin{abstract}
The weak decays of $\Lambda_{b} \rightarrow \Lambda+\gamma$ and $\Lambda_{b} \rightarrow \Lambda+l^{+} l^{-}$are investigated in the Standard Model using light-cone sum rules approach. The higher twist distribution amplitudes of $\Lambda$ baryon to the leading conformal spin are included in the sum rules for transition form factors. Our results indicate that the higher twist distribution amplitudes almost have no influences on the transition form factors retaining the heavy quark spin symmetry, while such corrections can result in significant impacts on the form factors breaking the heavy quark spin symmetry. Two phenomenological models (COZ and FZOZ) for the wave function of $\Lambda$ baryon are also employed in the sum rules for a comparison, which can give rise to the form factors approximately five times larger than that in terms of conformal expansion. Utilizing the form factors calculated in LCSR, the physical observables like decay rate, polarization asymmetry and forward-backward asymmetry are analyzed for the decays of $\Lambda_{b} \rightarrow \Lambda \gamma$, $\Lambda l^{+} l^{-}$.
\end{abstract}

\section{INTRODUCTION}

Generally, new physics can be accessible through rare decays, where the contributions from the Standard Model (SM) are suppressed enough. Hence, such decays can provide an ideal platform to test the SM precisely as well as to bound new physics parameters stringently. Rare decays involving $b \rightarrow s$ flavor changing neutral current (FCNC), which are forbidden at the tree level in the standard model, can only be induced by Glashow-Iliopoulos-Maiani mechanism [1] via loop diagrams. The Cabibbo-Kobayashi-Maskawa (CKM) matrix [2, 3] elements can be determined quantitatively from $b \rightarrow s$ rare decays, $B_{s}^{0}-\bar{B}_{s}^{0}$ mixing [4] etc., which will test its unitarity under the requirement of the SM.

It is well known that the inclusive decays are relatively robust theoretically, since the decay rate can be systematically and reasonably approximated by the decay of a free $b$ quark into light quarks, gluons and photons; but the counterpart of experimental measurements are quite difficult. On the 
contrary, the experimental investigations of exclusive decays are comparably easier; while the theoretical analysis of these modes encounter formidable difficulties due to lack of good understanding of QCD at low energy. As a matter of fact, there have been extensive studies on the exclusive decays of $B \rightarrow M l^{+} l^{-}[5,6,67,8,19,10], B \rightarrow V(A) \gamma$ [11, 12, 13, 14, 15, 16, 17, 18, 19, 20, 21] in the literature with varying degrees of theoretical rigor and emphasis. Unlike mesonic decays, the investigations of FCNC $b \rightarrow s$ transition for bottom baryonic decays $\Lambda_{b} \rightarrow \Lambda \gamma$ and $\Lambda_{b} \rightarrow \Lambda l^{+} l^{-}$are much behind because more degrees of freedom are involved in the bound state of baryon system at the quark level. It should be pointed out that such baryonic decays can offer the unique ground to extract the helicity structure of effective Hamiltonian for FCNC $b \rightarrow s$ transition in the SM and beyond, which is lost in the hadronization of meson case. From the viewpoint of experiment, the only drawback of bottom baryon decays is that the production rate of $\Lambda_{b}$ baryon in $b$ quark hadronization is about four times less than that of the $B$ meson.

The polarization asymmetry and forward-backward asymmetry in rare decays are very sensitive to the new physics effects beyond the SM [22, 23, 24, 25, 26, 27, 28, 29]. For instance, the polarization asymmetry of $\Lambda$ baryon in $\Lambda_{b} \rightarrow \Lambda+l^{+} l^{-}$decays is dependent heavily on the righthanded current [24], which is suppressed in the SM. However, before claiming the new physics signal, one should have a serious and comprehensive scrutiny of discrepancies between experimental data and theoretical predictions within the SM to confirm whether they are indeed due to contributions from new physics or missing effects in the SM, such as higher power and next-to-leading order corrections, soft gluon effects and so on.

Currently, there has been some studies in the literature on $\Lambda_{b} \rightarrow \Lambda$ transition form factors ranging from phenomenological models including the pole model (PM) 30], covariant oscillator quark model (COQM) 31], MIT bag model (BM) [32] and non-relativistic quark model [33] to QCD sum rule approach (QCDSR) [34] and also perturbative QCD (PQCD) approach [35]. It can be observed that the available theoretical predictions vary from each other and can differ even by the orders of magnitude. For instance, the decay width of $\Lambda_{b} \rightarrow \Lambda \gamma$ in PQCD approach is about one to two orders smaller than that obtained within other frameworks.

It is suggested that the soft non-perturbative contribution to the transition form factor can be calculated quantitatively in the framework of light-cone sum rules (LCSR) approach [36, 37, 38, 39, 40], which is a fully relativistic approach and well rooted in quantum field theory, in a systematic and almost model-independent way. As a marriage of standard QCDSR technique [41, 42, 43] and theory of hard exclusive process [44, 45, 46, 47, 48, 49, 50, 51], LCSR cure the problem of QCDSR applying to the large momentum transfer by performing the op- 
erator product expansion (OPE) in terms of twist of the revelent operators rather than their dimension [52]. Therefore, the principal discrepancy between QCDSR and LCSR consists in that non-perturbative vacuum condensates representing the long-distance quark and gluon interactions in the short-distance expansion are substituted by the light cone distribution amplitudes (LCDAs) describing the distribution of longitudinal momentum carried by the valence quarks of hadronic bound system in the expansion of transverse-distance between partons in the infinite momentum frame. Phenomenologically, LCSR has been widely applied to investigate the transition of mesons and baryons in the recent years, such as bottom meson decays [53, 54, 55, 56, 57, 58], electromagnetic form factors of nucleon [59, 60, 61], pion electroproduction [62], semi-leptonic weak decays of $\Lambda_{b} \rightarrow p l \nu_{l}$ [63], $\Lambda_{c} \rightarrow \Lambda l \nu_{l}$ [64], and can make consistent predictions with experimental data successfully. The generalization to the $\gamma^{*} N \rightarrow \Delta$ transition [65], $\Sigma \rightarrow N$ transition [66], radiative decays of decuplet to octet baryons [67] and meson-octet baryon couplings [68] have also been worked out.

LCDAs are the fundamental non-perturbative functions in the LCSR, which describes the hadronic structure in rare parton configurations with a fixed number of Fock components at small transverse separation in the infinite momentum frame. There have been continuous interests concentrating on the research of pre-asymptotic corrections to the distribution amplitudes of hadrons in the exclusive reactions over two decades, in an attempt to accommodate the experimental data, such as the electromagnetic form factors of pion and nucleon [51]. In particular, the LCDAs of mesons including higher twist have been investigated extensively [51, 69, 70, 71, 72, 73, 74, 75]. In contrast to the meticulous and intensive study of mesonic LCDAs, the corresponding task for the baryonic counterpart received less attention in the past due to the complexity of the inner structures for baryon. Fortunately, an exciting attempt to study the LCDAs of baryon systematically was carried out in 76] for the first time, where all the transverse degrees of freedom are integrated out and the higher twist distribution amplitudes contributed by the light-ray operators involving "minus" components of the quark field operators were investigated in detail. Subsequently, an alternative approach to classify the three-quark light-cone amplitudes of proton was put forward in [77, 78, 79], where the transverse degree of freedom of partons is retained and the minus component of field operators are eliminated in favor of the plus and transverse ones with the help of equations of motion. It is mentioned in Ref. [80] that both techniques are probably equivalent. In the present work, we would like to follow the prescription presented in [76] for LCSR approach to study the rare decay of $\Lambda_{b} \rightarrow \Lambda+\gamma$ and $\Lambda_{b} \rightarrow \Lambda+l^{+} l^{-}$, which can be also generalized to the decays of $\Lambda_{b}$ to heavier $\Lambda$ baryons [81, 82], once the distribution amplitudes of corresponding baryons are 
available.

The organization of this paper is as following: In section II, we collect the effective Hamiltonian responsible for FCNC transition of $b \rightarrow s \gamma$ and $b \rightarrow s l^{+} l^{-}$. Parameterizations of various hadronic matrix elements $\left\langle\Lambda\left|\bar{s} \Gamma_{i} b\right| \Lambda_{b}\right\rangle$ with $\Gamma_{i}$ being all the possible Lorentz structures are also presented here. Section III contains the derivation of LCSR for both $\Lambda_{b} \rightarrow \Lambda+\gamma$ and $\Lambda_{b} \rightarrow \Lambda+l^{+} l^{-}$decays up to the leading order of $\alpha_{s}$ with the corrections from higher twists. We find that the relations between various transition form factors existing in the heavy quark effective theory (HQET) and large energy effective theory (LEET) are well respected within the framework of LCSR approach. The numerical analysis of LCSR for transition form factors are displayed in section IV, where the corrections from the distribution amplitudes of higher twist and comparisons with the results that derived in other methods are investigated at length. In section V, we apply the available form factors to study the invariant mass distribution of lepton pair, $\Lambda$ polarization asymmetry and forward-backward asymmetry of $\Lambda_{b} \rightarrow \Lambda+l^{+} l^{-}$together with the decay width and $\Lambda$ polarization asymmetry of $\Lambda_{b} \rightarrow \Lambda \gamma$. We confirm the finding in Ref. [34, 83] that the $\Lambda$ polarization asymmetry of $\Lambda_{b} \rightarrow \Lambda \gamma$ is completely independent on the hadronic transition form factors, which makes it a good quantity to study the chiral structure of $b \rightarrow s$ effective Hamiltonian and probe the new physics beyond the SM without receiving any pollution from strong interactions. Numerical results of such observables and comparisons with that obtained in other theoretical methods, in particular QCDSR and PQCD approach, are also included in this section. Section VI is devoted to our conclusions.

\section{EFFECTIVE HAMILTONIAN AND PARAMETERIZATIONS OF MATRIX ELEMENT}

\section{A. Effective Hamiltonian for $b \rightarrow s$ transition}

Integrating out the particles including top quark, $W^{ \pm}$and $Z$ bosons above scale $\mu=O\left(m_{b}\right)$, we arrive at the effective Hamiltonian responsible for the $b \rightarrow s l^{+} l^{-}$transition [84]

$$
\begin{aligned}
H_{e f f}\left(b \rightarrow s l^{+} l^{-}\right)= & -\frac{G_{F}}{2 \sqrt{2}} V_{t b} V_{t s}^{*}\left[\sum_{i=1}^{6} C_{i}(\mu) Q_{i}(\mu)+C_{7 \gamma}(\mu) Q_{7 \gamma}(\mu)+C_{8 G}(\mu) Q_{8 G}(\mu)\right. \\
& \left.+C_{9}(\mu) Q_{9}(\mu)+C_{10}(\mu) Q_{10}(\mu)\right],
\end{aligned}
$$

where we have neglected the terms proportional to $V_{u b} V_{u s}^{*}$ on account of $\left|V_{u b} V_{u s}^{*} / V_{t b} V_{t s}^{*}\right|<0.02$ and the complete list of the operators can be found in [84]. In the SM, Wilson coefficients $C_{i}$ at scale 
TABLE I: Numerical values of Wilson coefficients in the NDR scheme at scale $\mu=m_{b}[10,22,34]$.

\begin{tabular}{c|c|c|c|c|c|c|c|c|c}
\hline \hline$C_{1}$ & $C_{2}$ & $C_{3}$ & $C_{4}$ & $C_{5}$ & $C_{6}$ & $C_{7 \gamma}$ & $C_{8 G}$ & $C_{9}$ & $C_{10}$ \\
\hline-0.218 & 1.107 & 0.011 & -0.026 & 0.007 & -0.031 & -0.305 & -0.15 & 4.344 & -4.669 \\
\hline \hline
\end{tabular}

$\mu=m_{b}$ calculated in the naive dimensional regularization (NDR) scheme are collected in Table I]

In terms of the Hamiltonian in Eq. (1), we can derive the free quark decay amplitude for $b \rightarrow s l^{+} l^{-}$process as

$$
\begin{aligned}
M\left(b \rightarrow s l^{+} l^{-}\right)= & \frac{G_{F}}{2 \sqrt{2}} V_{t b} V_{t s}^{*} \frac{\alpha_{e m}}{\pi}\left\{-\frac{2 i}{q^{2}} C_{7}^{e f f}(\mu) \bar{s} \sigma_{\mu \nu} q^{\nu}\left(m_{b} R+m_{s} L\right) b \bar{l} \gamma^{\mu} l\right. \\
& \left.+C_{9}^{e f f}(\mu) \bar{s} \gamma_{\mu} L b \bar{l} \gamma^{\mu} l+C_{10} \bar{s} \gamma_{\mu} L b \bar{l} \gamma^{\mu} \gamma_{5} l\right\}
\end{aligned}
$$

We should emphasize that the Wilson coefficient $C_{10}$ does not renormalize under QCD corrections and hence is independent on the energy scale $\mu \simeq O\left(m_{b}\right)$, since the operator $O_{10}$ can not be induced by the insertion of four-quark operators due to the absence of $Z$ boson in the effective theory. Moreover, the above quark decay amplitude can also receive additional contributions from the matrix element of four-quark operators, $\sum_{i=1}^{6}\left\langle l^{+} l^{-} s\left|O_{i}\right| b\right\rangle$, which are usually absorbed into the effective Wilson coefficient $C_{9}^{e f f}(\mu)$. To be more specific, we can decompose $C_{9}^{e f f}(\mu)$ into the following three parts [85, 86, 87, 88, 89, 90, 91]

$$
C_{9}^{e f f}(\mu)=C_{9}(\mu)+Y_{S D}\left(z, s^{\prime}\right)+Y_{L D}\left(z, s^{\prime}\right),
$$

where the parameters $z$ and $s^{\prime}$ are defined as $z=m_{c} / m_{b}, s^{\prime}=q^{2} / m_{b}^{2}$. $Y_{S D}\left(z, s^{\prime}\right)$ describes the short-distance contributions from four-quark operators far away from the $c \bar{c}$ resonance regions, which can be calculated reliably in perturbative theory. The long-distance contributions $Y_{L D}\left(z, s^{\prime}\right)$ from four-quark operators near the $c \bar{c}$ resonance cannot be calculated from first principles of QCD and are usually parameterized in the form of a phenomenological Breit-Wigner formula making use of the vacuum saturation approximation and quark-hadron duality. The manifest expressions for $Y_{S D}\left(z, s^{\prime}\right)$ and $Y_{L D}\left(z, s^{\prime}\right)$ can be written as [22, 23, 24, 25]

$$
\begin{aligned}
Y_{S D}\left(z, s^{\prime}\right)= & h\left(z, s^{\prime}\right)\left(3 C_{1}(\mu)+C_{2}(\mu)+3 C_{3}(\mu)+C_{4}(\mu)+3 C_{5}(\mu)+C_{6}(\mu)\right) \\
& -\frac{1}{2} h\left(1, s^{\prime}\right)\left(4 C_{3}(\mu)+4 C_{4}(\mu)+3 C_{5}(\mu)+C_{6}(\mu)\right) \\
& -\frac{1}{2} h\left(0, s^{\prime}\right)\left(C_{3}(\mu)+3 C_{4}(\mu)\right)+\frac{2}{9}\left(3 C_{3}(\mu)+C_{4}(\mu)+3 C_{5}(\mu)+C_{6}(\mu)\right),
\end{aligned}
$$




$$
\begin{aligned}
Y_{L D}\left(z, s^{\prime}\right)= & \frac{3}{\alpha_{e m}^{2}}\left(3 C_{1}(\mu)+C_{2}(\mu)+3 C_{3}(\mu)+C_{4}(\mu)+3 C_{5}(\mu)+C_{6}(\mu)\right) \\
& \sum_{j=\psi, \psi^{\prime}} \omega_{j}\left(q^{2}\right) k_{j} \frac{\pi \Gamma\left(j \rightarrow l^{+} l^{-}\right) M_{j}}{q^{2}-M_{j}^{2}+i M_{j} \Gamma_{j}^{t o t}}
\end{aligned}
$$

with

$$
\begin{aligned}
& h\left(z, s^{\prime}\right)=-\frac{8}{9} \ln z+\frac{8}{27}+\frac{4}{9} x-\frac{2}{9}(2+x)|1-x|^{1 / 2} \begin{cases}\ln \left|\frac{\sqrt{1-x}+1}{\sqrt{1-x}-1}\right|-i \pi & \text { for } x \equiv 4 z^{2} / s^{\prime}<1 \\
2 \arctan \frac{1}{\sqrt{x-1}} & \text { for } x \equiv 4 z^{2} / s^{\prime}>1\end{cases} \\
& h\left(0, s^{\prime}\right)=\frac{8}{27}-\frac{8}{9} \ln \frac{m_{b}}{\mu}-\frac{4}{9} \ln s^{\prime}+\frac{4}{9} i \pi .
\end{aligned}
$$

Here $M_{j}\left(\Gamma_{j}^{t o t}\right)$ are the masses (widths) of the intermediate resonant states and $\Gamma\left(j \rightarrow l^{+} l^{-}\right)$denote the partial decay width for the transition of vector charmonium state to massless lepton pair, which can be expressed in terms of the decay constant of charmonium through the relation [92]

$$
\Gamma\left(j \rightarrow l^{+} l^{-}\right)=\pi \alpha_{e m}^{2} \frac{16}{27} \frac{f_{j}^{2}}{M_{j}} .
$$

The phenomenological parameter $k_{j}$ in Eq.(5) is introduced to account for inadequacies of the factorization approximation, which can be determined from

$$
B R\left(\Lambda_{b} \rightarrow \Lambda J / \psi \rightarrow \Lambda l^{+} l^{-}\right)=B R\left(\Lambda_{b} \rightarrow \Lambda J / \psi\right) \cdot B R\left(J / \psi \rightarrow l^{+} l^{-}\right)
$$

The function $\omega_{j}\left(q^{2}\right)$ introduced in Eq.(5) is to compensate the naive treatment of long distance contributions due to the charm quark loop in the spirit of quark-hadron duality, which can overestimate the genuine effect of the charm quark at small $q^{2}$ remarkably ${ }^{1}$. The quantity $\omega_{j}\left(q^{2}\right)$ can be normalized to $\omega_{j}\left(M_{\psi_{j}}^{2}\right)=1$, but its exact form is unknown at present. It has been shown that there is a suppression for $\omega_{j}\left(q^{2}\right)$ when going down to $q^{2}=0$ with $\omega(0)<0.13$ (and could be even smaller) 98], hence we can neglect the resonant contributions for $b \rightarrow s \gamma$ safely. Since the dominant contribution of the resonances is in the vicinity of the intermediate $\psi_{i}$ masses, we will simply use $\omega_{j}\left(q^{2}\right)=1$ in our numerical calculations. Moreover, the non-factorizable effects 92, 93, 94, 99] from the charm loop can bring about further corrections to the radiative $b \rightarrow s \gamma$ transition, which can be absorbed into the effective Wilson coefficient $C_{7}^{e f f}$ as usual. Specifically, the Wilson coefficient $C_{7}^{e f f}$ is given by [22]

$$
C_{7}^{e f f}(\mu)=C_{7}(\mu)+C_{b \rightarrow s \gamma}^{\prime}(\mu)
$$

\footnotetext{
${ }^{1}$ For a more detailed discussion on long-distance and short-distance contributions from the charm loop, one can refer to references [10, 92, 93, 94, 95, 96, 97].
} 
with

$$
\begin{aligned}
C_{b \rightarrow s \gamma}^{\prime}(\mu) & =i \alpha_{s}\left[\frac{2}{9} \eta^{14 / 23}\left(G_{1}\left(x_{t}\right)-0.1687\right)-0.03 C_{2}(\mu)\right] \\
G_{1}(x) & =\frac{x\left(x^{2}-5 x-2\right)}{8(x-1)^{3}}+\frac{3 x^{2} \ln ^{2} x}{4(x-1)^{4}}
\end{aligned}
$$

where $\eta=\alpha_{s}\left(m_{W}\right) / \alpha_{s}(\mu), x_{t}=m_{t}^{2} / m_{W}^{2}, C_{b \rightarrow s \gamma}^{\prime}$ is the absorptive part for the $b \rightarrow s c \bar{c} \rightarrow s \gamma$ rescattering and we have dropped out the tiny contributions proportion to CKM sector $V_{u b} V_{u s}^{*}$.

Similarly, the free quark decay amplitude for $b \rightarrow s \gamma$ can be written as [84]:

$$
M(b \rightarrow s \gamma)=\frac{G_{F}}{2 \sqrt{2}} V_{t b} V_{t s}^{*} \frac{e}{4 \pi^{2}} C_{7}^{e f f}(\mu) \bar{s} \sigma_{\mu \nu} q^{\nu}\left(m_{b} R+m_{s} L\right) b F^{\mu \nu}
$$

We stress again that one should add a term

$$
\left[3 C_{1}(\mu)+C_{2}(\mu)+3 C_{3}(\mu)+C_{4}(\mu)+3 C_{5}(\mu)+C_{6}(\mu)\right] \frac{3}{\alpha_{e m}^{2}} \sum_{j=\psi, \psi^{\prime}} \omega_{j}(0) k_{j} \frac{\pi \Gamma\left(j \rightarrow l^{+} l^{-}\right) M_{j}}{q^{2}-M_{j}^{2}+i M_{j} \Gamma_{j}^{t o t}}(13)
$$

to the effective Wilson coefficient $C_{7}^{e f f}$, if the long-distance contributions from the charm quark loop in the resonance regions are included. As mentioned above, this type of effects are suppressed heavily both by the Breit-Wigner factor $\sim \Gamma_{i} / M_{i}$ and phenomenological parameter $\omega(0)$, and hence are neglected in the later discussions.

\section{B. Parameterizations of hadronic matrix element}

With the free quark decay amplitude available, we can proceed to calculate the decay amplitudes for $\Lambda_{b} \rightarrow \Lambda \gamma$ and $\Lambda_{b} \rightarrow \Lambda l^{+} l^{-}$at hadron level, which can be obtained by sandwiching the free quark amplitudes between the initial and final baryon states. Consequently, the following four hadronic matrix elements

$$
\begin{aligned}
\left\langle\Lambda(P)\left|\bar{s} \gamma_{\mu} b\right| \Lambda_{b}(P+q)\right\rangle, & \left\langle\Lambda(P)\left|\bar{s} \gamma_{\mu} \gamma_{5} b\right| \Lambda_{b}(P+q)\right\rangle \\
\left\langle\Lambda(P)\left|\bar{s} \sigma_{\mu \nu} b\right| \Lambda_{b}(P+q)\right\rangle, & \left\langle\Lambda(P)\left|\bar{s} \sigma_{\mu \nu} \gamma_{5} b\right| \Lambda_{b}(P+q)\right\rangle
\end{aligned}
$$


need to be computed as can be observed from Eqs. (22) and (12). Generally, the above four matrix elements can be parameterized in terms of a series of form factors as [22, 26, 27, 28, 29]

$$
\begin{aligned}
\left\langle\Lambda(P)\left|\bar{s} \gamma_{\mu} b\right| \Lambda_{b}(P+q)\right\rangle= & \bar{\Lambda}(P)\left(g_{1} \gamma_{\mu}+g_{2} i \sigma_{\mu \nu} q^{\nu}+g_{3} q_{\mu}\right) \Lambda_{b}(P+q), \\
\left\langle\Lambda(P)\left|\bar{s} \gamma_{\mu} \gamma_{5} b\right| \Lambda_{b}(P+q)\right\rangle= & \bar{\Lambda}(P)\left(G_{1} \gamma_{\mu}+G_{2} i \sigma_{\mu \nu} q^{\nu}+G_{3} q_{\mu}\right) \gamma_{5} \Lambda_{b}(P+q), \\
\left\langle\Lambda(P)\left|\bar{s} \sigma_{\mu \nu} b\right| \Lambda_{b}(P+q)\right\rangle= & \bar{\Lambda}(P)\left[h_{1} \sigma_{\mu \nu}-i h_{2}\left(\gamma_{\mu} q_{\nu}-\gamma_{\nu} q_{\mu}\right)\right. \\
& \left.-i h_{3}\left(\gamma_{\mu} P_{\nu}-\gamma_{\nu} P_{\mu}\right)-i h_{4}\left(P_{\mu} q_{\nu}-P_{\nu} q_{\mu}\right)\right] \Lambda_{b}(P+q), \\
\left\langle\Lambda(P)\left|\bar{s} \sigma_{\mu \nu} \gamma_{5} b\right| \Lambda_{b}(P+q)\right\rangle= & \bar{\Lambda}(P)\left[H_{1} \sigma_{\mu \nu}-i H_{2}\left(\gamma_{\mu} q_{\nu}-\gamma_{\nu} q_{\mu}\right)\right. \\
& \left.-i H_{3}\left(\gamma_{\mu} P_{\nu}-\gamma_{\nu} P_{\mu}\right)-i H_{4}\left(P_{\mu} q_{\nu}-P_{\nu} q_{\mu}\right)\right] \gamma_{5} \Lambda_{b}(P+q),
\end{aligned}
$$

where all the form factors $g_{i}, G_{i}, h_{i}$ and $H_{i}$ are functions of the square of momentum transfer $q^{2}$. Rewriting the Eqs. (17][18), we have the baryon matrix elements for the dipole operators as following

$$
\begin{aligned}
\left\langle\Lambda(P)\left|\bar{s} i \sigma_{\mu \nu} q^{\nu} b\right| \Lambda_{b}(P+q)\right\rangle & =\bar{\Lambda}(P)\left(f_{1} \gamma_{\mu}+f_{2} i \sigma_{\mu \nu} q^{\nu}+f_{3} q_{\mu}\right) \Lambda_{b}(P+q), \\
\left\langle\Lambda(P)\left|\bar{s} i \sigma_{\mu \nu} \gamma_{5} q^{\nu} b\right| \Lambda_{b}(P+q)\right\rangle & =\bar{\Lambda}(P)\left(F_{1} \gamma_{\mu}+F_{2} i \sigma_{\mu \nu} q^{\nu}+F_{3} q_{\mu}\right) \gamma_{5} \Lambda_{b}(P+q),
\end{aligned}
$$

with

$$
\begin{aligned}
f_{1} & =\frac{2 h_{2}-h_{3}+h_{4}\left(m_{\Lambda_{b}}+m_{\Lambda}\right)}{2} q^{2}, \\
f_{2} & =\frac{2 h_{1}+h_{3}\left(m_{\Lambda}-m_{\Lambda_{b}}\right)+h_{4} q^{2}}{2}, \\
f_{3} & =\frac{m_{\Lambda}-m_{\Lambda_{b}}}{q^{2}} f_{1}, \\
F_{1} & =\frac{2 H_{2}-H_{3}+H_{4}\left(m_{\Lambda_{b}}-m_{\Lambda}\right)}{2} q^{2}, \\
F_{2} & =\frac{2 H_{1}+H_{3}\left(m_{\Lambda}+m_{\Lambda_{b}}\right)+H_{4} q^{2}}{2}, \\
F_{3} & =\frac{m_{\Lambda}+m_{\Lambda_{b}}}{q^{2}} F_{1} .
\end{aligned}
$$

It should be emphasized that the form factors $f_{3}$ and $F_{3}$ do not contribute to the decay amplitude of $\Lambda_{b} \rightarrow \Lambda+l^{+} l^{-}$due to the conservation of vector current, namely $q^{\mu} \bar{l} \gamma_{\mu} l=0$. Concentrating on the radiative decay of $\Lambda_{b} \rightarrow \Lambda \gamma$, we then observe that the matrix element of magnetic penguin operators can be simplified as

$$
\begin{aligned}
\left\langle\Lambda(P)\left|\bar{s} i \sigma_{\mu \nu} q^{\nu} b\right| \Lambda_{b}(P+q)\right\rangle & =f_{2}(0) \bar{\Lambda}(P) i \sigma_{\mu \nu} q^{\nu} \Lambda_{b}(P+q), \\
\left\langle\Lambda(P)\left|\bar{s} i \sigma_{\mu \nu} \gamma_{5} q^{\nu} b\right| \Lambda_{b}(P+q)\right\rangle & =F_{2}(0) \bar{\Lambda}(P) i \sigma_{\mu \nu} \gamma_{5} q^{\nu} \Lambda_{b}(P+q) .
\end{aligned}
$$


For the completeness, we also present the parameterizations of matrix elements involving the scalar $\bar{s} b$ and pseudo-scalar $\bar{s} \gamma_{5} b$ currents, which can be obtained from the Eqs. (15) and (16) by contracting both sides to the four-momentum $q^{\mu}$

$$
\begin{aligned}
\left\langle\Lambda(P)|\bar{s} b| \Lambda_{b}(P+q)\right\rangle & =\frac{1}{m_{b}+m_{s}} \bar{\Lambda}(P)\left[g_{1}\left(m_{\Lambda_{b}}-m_{\Lambda}\right)+g_{3} q^{2}\right] \Lambda_{b}(P+q), \\
\left\langle\Lambda(P)\left|\bar{s} \gamma_{5} b\right| \Lambda_{b}(P+q)\right\rangle & =\frac{1}{m_{b}-m_{s}} \bar{\Lambda}(P)\left[G_{1}\left(m_{\Lambda_{b}}+m_{\Lambda}\right)-G_{3} q^{2}\right] \gamma_{5} \Lambda_{b}(P+q) .
\end{aligned}
$$

The two independent form factors $\xi_{1}$ and $\xi_{2}$ in HQET are defined as

$$
\left\langle\Lambda(P)|\bar{b} \Gamma s| \Lambda_{b}(P+q)\right\rangle=\bar{\Lambda}(P)\left[\xi_{1}\left(q^{2}\right)+\not\left\langle\xi_{2}\left(q^{2}\right)\right] \Gamma \Lambda_{b}(P+q),\right.
$$

with $\Gamma$ being an arbitrary Lorentz structure and $v_{\mu}$ being the four-velocity of $\Lambda_{b}$ baryon. Comparing Eqs. (15, 16), (19,20) and the Eq. (31), one can easily find [22, 26, 27, 28, 29]

$$
\begin{aligned}
& f_{1}=F_{1}=\frac{q^{2}}{m_{\Lambda_{b}}} \xi_{2}, \\
& f_{2}=F_{2}=g_{1}=G_{1}=\xi_{1}+\frac{m_{\Lambda}}{m_{\Lambda_{b}}} \xi_{2}, \\
& f_{3}=\frac{m_{\Lambda}-m_{\Lambda_{b}}}{m_{\Lambda_{b}}} \xi_{2}, \\
& F_{3}=\frac{m_{\Lambda}+m_{\Lambda_{b}}}{m_{\Lambda_{b}}} \xi_{2}, \\
& g_{2}=G_{2}=g_{3}=G_{3}=\frac{\xi_{2}}{m_{\Lambda_{b}}} .
\end{aligned}
$$

It is known that Eq. (31) is successful at zero recoil region (with large $q^{2}$ ) in the heavy quark limit. As for the large recoil region, the large energy effective theory implies that the form factors are independent of both energy of light hadron $(E)$ and the heavy quark mass $\left(m_{b}\right)$ with the assumption of Feynman mechanism, which indicates that Eq. (31) is still well defined owing to the tiny effects from $1 / m_{b}, 1 / E$ and $\alpha_{s}$ corrections [100]. 


\section{LIGHT-CONE SUM RULES FOR THE TRANSITION FORM FACTORS}

The most general decomposition of matrix element for three-quark operator with light-like separations $x^{2} \rightarrow 0$ between vacuum and $\Lambda$ baryon state at tree level can be written as [76]

$$
\begin{aligned}
& 4\left\langle 0\left|\epsilon^{i j k} u_{\alpha}^{i}\left(a_{1} x\right) d_{\beta}^{j}\left(a_{2} x\right) s_{\gamma}^{k}\left(a_{3} x\right)\right| \Lambda(P)\right\rangle \\
& =\mathcal{S}_{1} m_{\Lambda} C_{\alpha \beta}\left(\gamma_{5} \Lambda\right)_{\gamma}+\mathcal{S}_{2} m_{\Lambda}^{2} C_{\alpha \beta}\left(\not x \gamma_{5} \Lambda\right)_{\gamma}+\mathcal{P}_{1} m_{\Lambda}\left(\gamma_{5} C\right)_{\alpha \beta} \Lambda_{\gamma}+\mathcal{P}_{2} m_{\Lambda}^{2}\left(\gamma_{5} C\right)_{\alpha \beta}(\not x \Lambda)_{\gamma} \\
& +\mathcal{V}_{1}(P C)_{\alpha \beta}\left(\gamma_{5} \Lambda\right)_{\gamma}+\mathcal{V}_{2} m_{\Lambda}(P C)_{\alpha \beta}\left(\not x \gamma_{5} \Lambda\right)_{\gamma}+\mathcal{V}_{3} m_{\Lambda}\left(\gamma_{\mu} C\right)_{\alpha \beta}\left(\gamma^{\mu} \gamma_{5} \Lambda\right)_{\gamma} \\
& +\mathcal{V}_{4} m_{\Lambda}^{2}(\not x C)_{\alpha \beta}\left(\gamma_{5} \Lambda\right)_{\gamma}+\mathcal{V}_{5} m_{\Lambda}^{2}\left(\gamma_{\mu} C\right)_{\alpha \beta}\left(i \sigma^{\mu \nu} x_{\nu} \gamma_{5} \Lambda\right)_{\gamma}+\mathcal{V}_{6} m_{\Lambda}^{3}(\not x C)_{\alpha \beta}\left(\not x \gamma_{5} \Lambda\right)_{\gamma} \\
& +\mathcal{A}_{1}\left(P \gamma_{5} C\right)_{\alpha \beta} \Lambda_{\gamma}+\mathcal{A}_{2} m_{\Lambda}\left(P \gamma_{5} C\right)_{\alpha \beta}(\not x \Lambda)_{\gamma}+\mathcal{A}_{3} m_{\Lambda}\left(\gamma_{\mu} \gamma_{5} C\right)_{\alpha \beta}\left(\gamma^{\mu} \Lambda\right)_{\gamma} \\
& +\mathcal{A}_{4} m_{\Lambda}^{2}\left(\not x \gamma_{5} C\right)_{\alpha \beta} \Lambda_{\gamma}+\mathcal{A}_{5} m_{\Lambda}^{2}\left(\gamma_{\mu} \gamma_{5} C\right)_{\alpha \beta}\left(i \sigma^{\mu \nu} x_{\nu} \Lambda\right)_{\gamma}+\mathcal{A}_{6} m_{\Lambda}^{3}\left(\not \gamma_{5} C\right)_{\alpha \beta}(\not x \Lambda)_{\gamma} \\
& +\mathcal{T}_{1}\left(P^{\nu} i \sigma_{\mu \nu} C\right)_{\alpha \beta}\left(\gamma^{\mu} \gamma_{5} \Lambda\right)_{\gamma}+\mathcal{T}_{2} m_{\Lambda}\left(x^{\mu} P^{\nu} i \sigma_{\mu \nu} C\right)_{\alpha \beta}\left(\gamma_{5} \Lambda\right)_{\gamma}+\mathcal{T}_{3} m_{\Lambda}\left(\sigma_{\mu \nu} C\right)_{\alpha \beta}\left(\sigma^{\mu \nu} \gamma_{5} \Lambda\right)_{\gamma} \\
& +\mathcal{T}_{4} m_{\Lambda}\left(P^{\nu} \sigma_{\mu \nu} C\right)_{\alpha \beta}\left(\sigma^{\mu \rho} x_{\rho} \gamma_{5} \Lambda\right)_{\gamma}+\mathcal{T}_{5} m_{\Lambda}^{2}\left(x^{\nu} i \sigma_{\mu \nu} C\right)_{\alpha \beta}\left(\gamma^{\mu} \gamma_{5} \Lambda\right)_{\gamma} \\
& +\mathcal{T}_{6} m_{\Lambda}^{2}\left(x^{\mu} P^{\nu} i \sigma_{\mu \nu} C\right)_{\alpha \beta}\left(\not x \gamma_{5} \Lambda\right)_{\gamma}+\mathcal{T}_{7} m_{\Lambda}^{2}\left(\sigma_{\mu \nu} C\right)_{\alpha \beta}\left(\sigma^{\mu \nu} \not x \gamma_{5} \Lambda\right)_{\gamma} \\
& +\mathcal{T}_{8} m_{\Lambda}^{3}\left(x^{\nu} \sigma_{\mu \nu} C\right)_{\alpha \beta}\left(\sigma^{\mu \rho} x_{\rho} \gamma_{5} \Lambda\right)_{\gamma},
\end{aligned}
$$

in view of the Lorentz covariance, spin and parity of $\Lambda$ baryon. Here, each of the 24 invariant functions $\mathcal{F}_{i},(\mathcal{F}=\mathcal{S}, \mathcal{P}, \mathcal{V}, \mathcal{A}, \mathcal{T})$ is a function of the scalar product $P \cdot x$ and the parameters $a_{i}$ denote coordinates of valence quarks.

The "calligraphic" functions $\mathcal{F}_{i}$ defined above do not have a definite twist. Decomposing the quark field operators into "plus" and "minus" components [76] and utilizing the equation of motion, the invariant function $\mathcal{F}_{i}$ can be expressed by the distribution amplitudes $A_{i}$ [64]:

$$
\begin{gathered}
\mathcal{A}_{1}=A_{1}, \quad 2 p \cdot x \mathcal{A}_{2}=-A_{1}+A_{2}-A_{3}, \\
2 \mathcal{A}_{3}=A_{3}, \quad 4 p \cdot x \mathcal{A}_{4}=-2 A_{1}-A_{3}-A_{4}+2 A_{5}, \\
4 p \cdot x \mathcal{A}_{5}=A_{3}-A_{4}, \quad(2 p \cdot x)^{2} \mathcal{A}_{6}=A_{1}-A_{2}+A_{3}+A_{4}-A_{5}+A_{6},
\end{gathered}
$$

where the twists of distribution amplitudes $A_{i}(i=1, \ldots, 6)$ can be easily determined by power countering as collected in Table II. Only the the relations associated with axial-vector distribution amplitudes $A_{i}$ of $\Lambda$ baryon are involved in the sum rules of form factors responsible for $\Lambda_{b} \rightarrow \Lambda$ transition, since the spectator quarks $[u d]$ in the $\Lambda_{b}$ baryon maintain their position at the origin up to the accuracy of leading order in $\alpha_{s}$.

Generally, the distribution amplitudes depend on the renormalization scale and can be expanded in contributions of light-ray operators with increasing conformal spin, where the constraints on 
TABLE II: Twist classification [76] of the distribution amplitudes given in Eq. (38).

\begin{tabular}{lllll}
\hline \hline & twist-3 & twist-4 & twist-5 & twist-6 \\
axial-vector & $A_{1}$ & $A_{2}, A_{3}$ & $A_{4}, A_{5}$ & $A_{6}$ \\
\hline \hline
\end{tabular}

operator mixing and equation of motion owing to conformal symmetry of the QCD Lagrangian 101] are also taken into account. To the leading logarithmic accuracy, the different conformal partial waves do not mix with each other, since the renormalization group (RG) are driven by tree-level counter terms and the conformal symmetry is well respected. Besides, it is known that QCDSR tends to overestimate the matrix elements corresponding to the higher conformal spin operators considerably from the experience of both calculating the pion electromagnetic form factor [102, 103] and nucleon form factor [59]. Therefore, following the Ref. [64], we would like to adopt the distribution amplitudes of $\Lambda$ baryon to the leading conformal spin accuracy in the present work.

The explicit forms of distribution amplitudes for $\Lambda$ baryon to the accuracy of leading conformal spin can be expressed as [64]

$$
\begin{aligned}
& A_{1}\left(x_{1}, x_{2}, x_{3}\right)=-120 x_{1} x_{2} x_{3} \phi_{3}^{0}, \\
& A_{2}\left(x_{1}, x_{2}, x_{3}\right)=-24 x_{1} x_{2} \phi_{4}^{0}, \\
& A_{3}\left(x_{1}, x_{2}, x_{3}\right)=-12 x_{3}\left(1-x_{3}\right) \psi_{4}^{0}, \\
& A_{4}\left(x_{1}, x_{2}, x_{3}\right)=-3\left(1-x_{3}\right) \phi_{5}^{0}, \\
& A_{5}\left(x_{1}, x_{2}, x_{3}\right)=-6 x_{3} \phi_{5}^{0}, \\
& A_{6}\left(x_{1}, x_{2}, x_{3}\right)=-2 \phi_{6}^{0},
\end{aligned}
$$

with

$$
\phi_{3}^{0}=\phi_{6}^{0}=-f_{\Lambda}, \quad \phi_{4}^{0}=\phi_{5}^{0}=-\frac{f_{\Lambda}+\lambda_{1}}{2}, \quad \psi_{4}^{0}=\psi_{5}^{0}=\frac{f_{\Lambda}-\lambda_{1}}{2}
$$

The estimations of non-perturbative parameters $f_{\Lambda}$ and $\lambda_{1}$ in the framework of QCDSR approach have been presented in Ref. [64] and to make the paper self contained, these are collected in the Appendix A.

With the LCDAs of $\Lambda$ baryon available, we are now in a position to derive the sum rules of transition form factors which are responsible for $\Lambda_{b} \rightarrow \Lambda \gamma$ and $\Lambda_{b} \rightarrow \Lambda l^{+} l^{-}$decays. The basic object in LCSR approach is the correlation function where one of the hadron is represented by the interpolating current with proper quantum number, such as spin, isospin, (charge) parity and 
so on; and the other is described by its vector state manifestly. Information on the hadronic transition form factor can be extracted by matching the Green function calculated in two different representations, i.e., phenomenological and theoretical forms, with the help of dispersion relation under the assumption of quark-hadron duality.

\section{A. Light-cone sum rules for $\Lambda_{b} \rightarrow \Lambda+\gamma$}

We consider the correlation function associating with $\Lambda_{b} \rightarrow \Lambda+\gamma$ decay determined by the matrix element

$$
z^{\nu} T_{\nu}(P, q)=i z^{\nu} \int d^{4} x e^{-i q \cdot x}\left\langle 0\left|T\left\{j_{\Lambda_{b}}(0) j_{\nu}(x)\right\}\right| \Lambda(P)\right\rangle
$$

between the vacuum and $\Lambda$ baryon state $|\Lambda(P)\rangle$. Generally, choice of interpolating current for baryon, in particular the light baryon, is not unique [104, 105, 106, 107, 108, 109]. The interpolating field $j_{\Lambda_{b}}(0)$ denoting the $\Lambda_{b}$ baryon and the transition current $j_{\nu}(x)$ describing the transition of $\Lambda_{b} \rightarrow \Lambda \gamma$ are given by

$$
\begin{aligned}
j_{\Lambda_{b}}(0) & =\epsilon^{i j k}\left[u^{i}(0) C \gamma_{5} \not d^{j}(0)\right] \not b^{k}(0), \\
j_{\nu}(x) & =i \bar{b}(x) \sigma_{\mu \nu}\left(1-\gamma_{5}\right) q^{\mu} s(x)
\end{aligned}
$$

In addition, the vacuum-to-baryon matrix element for the interpolating current can be parameterized as follows

$$
\left\langle 0\left|j_{\Lambda_{b}}(0)\right| \Lambda_{b}\left(P^{\prime}\right)\right\rangle=f_{\Lambda_{b}}\left(z \cdot P^{\prime}\right) \not \Lambda_{b}\left(P^{\prime}\right)
$$

Inserting the complete set of states between the currents in Eq. (41) with the same quantum numbers as $\Lambda_{b}$, we can arrive at the hadronic representation of the correlator

$$
z^{\nu} T_{\nu}=\frac{\left\langle 0\left|j_{\Lambda_{b}}\right| \Lambda_{b}\left(P^{\prime}\right)\right\rangle\left\langle\Lambda_{b}\left(P^{\prime}\right)\left|z^{\nu} j_{\nu}\right| \Lambda(P)\right\rangle}{m_{\Lambda_{b}}^{2}-P^{\prime 2}}+\sum_{h} \frac{\left\langle 0\left|j_{\Lambda_{b}}\right| h\left(P^{\prime}\right)\right\rangle\left\langle h\left(P^{\prime}\right)\left|z^{\nu} j_{\nu}\right| \Lambda(P)\right\rangle}{m_{h}^{2}-P^{\prime 2}},
$$

where $P^{\prime}=P+q$ and we have separated the contributions from the ground state and higher states corresponding to the $\Lambda_{b}$ baryon channel. Combining the Eq. (19, 201), (43, 44) and summing over the polarization of $\Lambda_{b}$ baryon, we can obtain the phenomenological representations of correlation function as

$$
z^{\nu} T_{\nu}=2 f_{\Lambda_{b}} \frac{\left(z \cdot P^{\prime}\right)^{2}}{m_{\Lambda_{b}}^{2}-P^{\prime 2}}\left[-f_{1} \not z+f_{2} \not \prec \not q-F_{1} \not \not \gamma_{5}-F_{2} \not \not \not q \gamma_{5}\right] \Lambda(P)+\ldots,
$$

where the ellipses stand for the terms proportional to the higher power of $1 / P$ in the infinite momentum kinematics $P \sim \infty, q \sim$ const, $z \sim 1 / P$ and the contributions from the higher states 


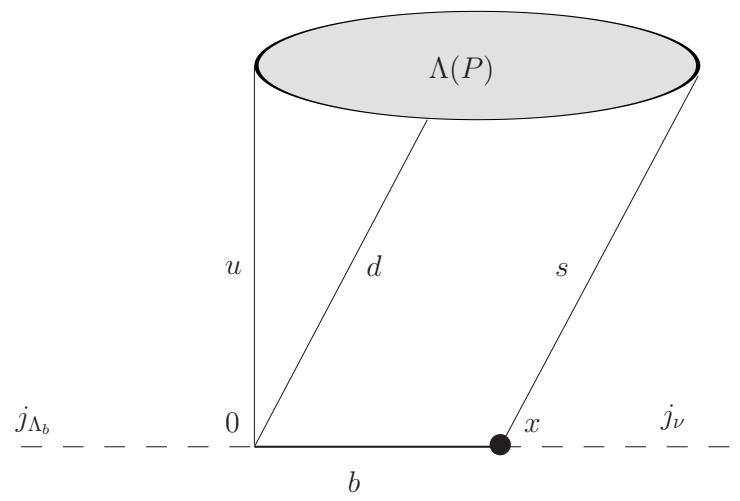

FIG. 1: The tree level contribution to the correlation function (41), where the thick solid line represents the heavy $b$ quark.

of $\Lambda_{b}$ channel. The advantage of contracting the correlation function with $z^{\nu}$ is that the Lorentz structures can be simplified owing to the null contributions from $z_{\nu}$ on the light-cone.

On the theoretical side, the correlation function (41) can also be computed in the perturbation theory with the help of OPE technique at the deep Euclidean region: $P^{\prime 2}, q^{2}=-Q^{2} \ll 0$. To the leading order of $\alpha_{s}$, the correlation function can be calculated by contracting the bottom quark field in Eq. (41) and inserting the free $b$ quark propagator

$$
z^{\nu} T_{\nu}=-2\left(C \gamma_{5} \not \not\right)_{\alpha \beta}\left[\not z\left(1-\gamma_{5}\right)\right]_{\gamma} \int d^{4} x \int \frac{d^{4} k}{(2 \pi)^{4}} e^{i(k-q) \cdot x} \frac{z \cdot k}{k^{2}-m_{b}^{2}}\left\langle 0\left|\epsilon^{i j k} u_{\alpha}^{i}(0) d_{\beta}^{j}(0) s_{\gamma}^{k}(x)\right| \Lambda(P)\right\rangle
$$

shown in Fig. 1]

The full quark propagator also receives corrections from the background field [110, 111] and can be written as

$$
\begin{aligned}
\left\langle 0\left|T\left\{b_{i}(x) \bar{b}_{j}(0)\right\}\right| 0\right\rangle= & \delta_{i j} \int \frac{d^{4} k}{(2 \pi)^{4}} e^{-i k x} \frac{i}{\not k-m_{b}}-i g \int \frac{d^{4} k}{(2 \pi)^{4}} e^{-i k x} \int_{0}^{1} d v\left[\frac{1}{2} \frac{\not k+m_{b}}{\left(m_{b}^{2}-k^{2}\right)^{2}} G_{i j}^{\mu \nu}(v x) \sigma_{\mu \nu}\right. \\
& \left.+\frac{1}{m_{b}^{2}-k^{2}} v x_{\mu} G^{\mu \nu}(v x) \gamma_{\nu}\right]
\end{aligned}
$$

where the first term is the free-quark propagator and $G_{i j}^{\mu \nu}=G_{\mu \nu}^{a} T_{i j}^{a}$ with $\operatorname{Tr}\left[T^{a} T^{b}\right]=\frac{1}{2} \delta^{a b}$. Inserting the second term proportional to the gluon field strength into the correlation function can result in the distribution amplitudes corresponding to the higher Fock states of $\Lambda$ baryon. It is expected that such corrections associating with the LCDAs of higher Fock states do not play any significant role in the sum rules for transition form factors [112], and therefore can be neglected safely in the presented work.

Substituting Eq. (37) into Eq. (46) and performing the integral in the coordinate space, we can 
achieve the correlation function in the momentum representation at the quark level as

$$
\begin{aligned}
z^{\nu} T_{\nu}=-2(z \cdot P)^{2} \not k \not\left(1-\gamma_{5}\right) \Lambda(P)\left\{\int \mathcal{D} x \frac{x_{3} A_{1}\left(x_{i}\right)}{\left(x_{3} P+q\right)^{2}-m_{b}^{2}}\right. \\
+M^{2} \int_{0}^{1} d x_{3} x_{3}^{2} \frac{-2 \tilde{A}_{1}\left(x_{3}\right)+\tilde{A}_{2}\left(x_{3}\right)-\tilde{A}_{3}\left(x_{3}\right)-\tilde{A}_{4}\left(x_{3}\right)+\tilde{A}_{5}\left(x_{3}\right)}{\left[\left(x_{3} P+q\right)^{2}-m_{b}^{2}\right]^{2}} \\
\left.\quad+2 M^{4} \int_{0}^{1} d x_{3} x_{3}^{3} \frac{\tilde{\tilde{A}}_{1}\left(x_{3}\right)-\tilde{\tilde{A}}_{2}\left(x_{3}\right)+\tilde{\tilde{A}}_{3}\left(x_{3}\right)+\tilde{\tilde{A}}_{4}\left(x_{3}\right)-\tilde{\tilde{A}}_{5}\left(x_{3}\right)+\tilde{\tilde{A}}_{6}\left(x_{3}\right)}{\left[\left(x_{3} P+q\right)^{2}-m_{b}^{2}\right]^{3}}\right\} \\
+2 q^{2}(z \cdot P)^{2} \not k\left(1+\gamma_{5}\right) \Lambda(P)\left\{M \int_{0}^{1} d x_{3} x_{3} \frac{-\tilde{A}_{1}\left(x_{3}\right)+\tilde{A}_{2}\left(x_{3}\right)-\tilde{A}_{3}\left(x_{3}\right)}{\left[\left(x_{3} P+q\right)^{2}-m_{b}^{2}\right]^{2}}\right. \\
\left.+2 M^{3} \int_{0}^{1} d x_{3} x_{3}^{2} \frac{\tilde{\tilde{A}}_{1}\left(x_{3}\right)-\tilde{\tilde{A}}_{2}\left(x_{3}\right)+\tilde{\tilde{A}}_{3}\left(x_{3}\right)+\tilde{\tilde{A}}_{4}\left(x_{3}\right)-\tilde{\tilde{A}}_{5}\left(x_{3}\right)+\tilde{\tilde{A}}_{6}\left(x_{3}\right)}{\left[\left(x_{3} P+q\right)^{2}-m_{b}^{2}\right]^{3}}\right\}+\ldots .
\end{aligned}
$$

where subleading terms in the infinite momentum frame kinematics denoted by the ellipses are not included. The distribution amplitudes with tildes are defined as

$$
\begin{aligned}
& \tilde{F}\left(x_{3}\right)=\int_{1}^{x_{3}} d x_{3}^{\prime} \int_{0}^{1-x_{3}^{\prime}} d x_{1} F\left(x_{1}, 1-x_{1}-x_{3}^{\prime}, x_{3}^{\prime}\right), \\
& \tilde{\tilde{F}}\left(x_{3}\right)=\int_{1}^{x_{3}} d x_{3}^{\prime} \int_{1}^{x_{3}^{\prime}} d x_{3}^{\prime \prime} \int_{0}^{1-x_{3}^{\prime}} d x_{1} F\left(x_{1}, 1-x_{1}-x_{3}^{\prime \prime}, x_{3}^{\prime \prime}\right),
\end{aligned}
$$

originating from the partial integral in the variable $x_{3}$ in order to eliminate the factor $1 / P \cdot x$ due to the insertion of distribution amplitudes in Eq. (38).

For the convenience of matching the correlation in QCD representation and hadronic level, the Eq. (48) is usually written in a form of dispersion integral as

$$
z^{\nu} T_{\nu}=(z \cdot P)^{2} \int_{m_{b}^{2}}^{\infty} d s \frac{\rho_{V}\left(s, Q^{2}\right) \not k\left(1-\gamma_{5}\right)+\rho_{T}\left(s, Q^{2}\right) \not k \not q\left(1-\gamma_{5}\right)}{s-P^{\prime 2}} \Lambda(P)+\ldots,
$$

where $Q^{2}$ is defined as $Q^{2}=-q^{2}$. With the assumption of quark-hadron duality, the higher states in the $\Lambda_{b}$ channel can be given by the same dispersion integral only with the lower bound replaced by the effective threshold parameter $s_{0}$. Besides, the Borel transformation is commonly introduced in the standard procedure of sum rules approach for the sake of compensating the deficiency due to the approximation of quark-hadron duality. Finally, we can obtain the sum rules for the transition 
form factors of the $\Lambda_{b} \rightarrow \Lambda \gamma$ decay as

$$
\begin{aligned}
f_{\Lambda_{b}} f_{1}\left(q^{2}\right) e^{-m_{\Lambda_{b}}^{2} / M_{B}^{2}}= & -q^{2} M\left\{\int _ { x _ { 0 } } ^ { 1 } \frac { d x _ { 3 } } { x _ { 3 } } e ^ { - s / M _ { B } ^ { 2 } } \left[\frac{1}{M_{B}^{2}}\left(-\tilde{A}_{1}\left(x_{3}\right)+\tilde{A}_{2}\left(x_{3}\right)-\tilde{A}_{3}\left(x_{3}\right)\right)\right.\right. \\
& \left.-\frac{M^{2}}{M_{B}^{4}}\left(\tilde{\tilde{A}}_{1}\left(x_{3}\right)-\tilde{\tilde{A}}_{2}\left(x_{3}\right)+\tilde{\tilde{A}}_{3}\left(x_{3}\right)+\tilde{\tilde{A}}_{4}\left(x_{3}\right)-\tilde{\tilde{A}}_{5}\left(x_{3}\right)+\tilde{\tilde{A}}_{6}\left(x_{3}\right)\right)\right] \\
& +\frac{x_{0} e^{-s_{0} / M_{B}^{2}}}{m_{b}^{2}-q^{2}+x_{0}^{2} M^{2}}\left[\left(-\tilde{A}_{1}\left(x_{3}\right)+\tilde{A}_{2}\left(x_{3}\right)-\tilde{A}_{3}\left(x_{3}\right)\right)\right. \\
& -\frac{M^{2}}{M_{B}^{2}}\left(\tilde{\tilde{A}}_{1}\left(x_{0}\right)-\tilde{\tilde{A}}_{2}\left(x_{0}\right)+\tilde{\tilde{A}}_{3}\left(x_{0}\right)+\tilde{\tilde{A}}_{4}\left(x_{0}\right)-\tilde{\tilde{A}}_{5}\left(x_{0}\right)+\tilde{\tilde{A}}_{6}\left(x_{0}\right)\right) \\
& \left.\left.+M^{2} x_{0} \frac{d}{d x_{0}}\left(\frac{x_{0}\left(\tilde{\tilde{A}}_{1}\left(x_{0}\right)-\tilde{\tilde{A}}_{2}\left(x_{0}\right)+\tilde{\tilde{A}}_{3}\left(x_{0}\right)+\tilde{\tilde{A}}_{4}\left(x_{0}\right)-\tilde{\tilde{A}}_{5}\left(x_{0}\right)+\tilde{\tilde{A}}_{6}\left(x_{0}\right)\right)}{m_{0}^{2}-q^{2}+x_{0}^{2} M^{2}}\right)\right]\right\}
\end{aligned}
$$

and

$$
\begin{aligned}
f_{\Lambda_{b}} f_{2}\left(q^{2}\right) e^{-m_{\Lambda_{b}}^{2} / M_{B}^{2}}= & \int_{x_{0}}^{1} d x_{3} e^{-s / M_{B}^{2}}\left[\left(\int_{0}^{1-x_{3}} d x_{1} A_{1}\left(x_{1}, 1-x_{1}-x_{3}, x_{3}\right)\right)\right. \\
& -\frac{M^{2}}{M_{B}^{2}}\left(-2 \tilde{A}_{1}\left(x_{3}\right)+\tilde{A}_{2}\left(x_{3}\right)-\tilde{A}_{3}\left(x_{3}\right)-\tilde{A}_{4}\left(x_{3}\right)+\tilde{A}_{5}\left(x_{3}\right)\right) \\
& \left.+\frac{M^{4}}{M_{B}^{4}}\left(\tilde{\tilde{A}}_{1}\left(x_{3}\right)-\tilde{\tilde{A}}_{2}\left(x_{3}\right)+\tilde{\tilde{A}}_{3}\left(x_{3}\right)+\tilde{\tilde{A}}_{4}\left(x_{3}\right)-\tilde{\tilde{A}}_{5}\left(x_{3}\right)+\tilde{\tilde{A}}_{6}\left(x_{3}\right)\right)\right] \\
& -\frac{M^{2} x_{0}^{2} e^{-s_{0} / M_{B}^{2}}}{m_{b}^{2}-q^{2}+x_{0}^{2} M^{2}}\left[\left(-2 \tilde{A}_{1}\left(x_{0}\right)+\tilde{A}_{2}\left(x_{0}\right)-\tilde{A}_{3}\left(x_{0}\right)-\tilde{A}_{4}\left(x_{0}\right)+\tilde{A}_{5}\left(x_{0}\right)\right)\right. \\
& -\frac{M^{2}}{M_{B}^{2}}\left(\tilde{\tilde{A}}_{1}\left(x_{0}\right)-\tilde{\tilde{A}}_{2}\left(x_{0}\right)+\tilde{\tilde{A}}_{3}\left(x_{0}\right)+\tilde{\tilde{A}}_{4}\left(x_{0}\right)-\tilde{\tilde{A}}_{5}\left(x_{0}\right)+\tilde{\tilde{A}}_{6}\left(x_{0}\right)\right)(52) \\
+ & \left.M^{2} \frac{d}{d x_{0}}\left(\frac{x_{0}^{2}\left(\tilde{\tilde{A}}_{1}\left(x_{0}\right)-\tilde{\tilde{A}}_{2}\left(x_{0}\right)+\tilde{\tilde{A}}_{3}\left(x_{0}\right)+\tilde{\tilde{A}}_{4}\left(x_{0}\right)-\tilde{\tilde{A}}_{5}\left(x_{0}\right)+\tilde{\tilde{A}}_{6}\left(x_{0}\right)\right)}{m_{0}^{2}-q^{2}+x_{0}^{2} M^{2}}\right)\right],
\end{aligned}
$$

with

$$
x_{0}=\frac{\sqrt{\left(Q^{2}+s_{0}-M^{2}\right)^{2}+4 M^{2}\left(Q^{2}+m_{b}^{2}\right)}-\left(Q^{2}+s_{0}-M^{2}\right)}{2 M^{2}} .
$$

It can be easily observed that the sum rules for form factors $F_{1}\left(q^{2}\right)$ and $F_{2}\left(q^{2}\right)$ are the same as that for $f_{1}\left(q^{2}\right)$ and $f_{2}\left(q^{2}\right)$ respectively, namely, $F_{1}\left(q^{2}\right)=f_{1}\left(q^{2}\right), F_{2}\left(q^{2}\right)=f_{2}\left(q^{2}\right)$. In addition, we indeed find that form factors $f_{2}\left(q^{2}\right)$ and $F_{2}\left(q^{2}\right)$ are exactly equal to zero at zero momentum transfer, which indicates that they do not play any role to the radiative decay $\Lambda_{b} \rightarrow \Lambda+\gamma$.

Now we are going to investigate the asymptotic behavior of form factors $f_{1}\left(q^{2}\right)$ and $f_{2}\left(q^{2}\right)$ in the limit of $Q^{2} \rightarrow \infty$. The form factors are dominated by the configurations with the recoiled $s$ quark taking most of the momentum of $\Lambda$ baryon. Employing the asymptotic distribution amplitudes of $\Lambda$ baryon and expanding the sum rules given in Eqs. (51) and (52) in $1 / Q^{2}$, we can get the 
following expressions of form factors in the limit of large momentum transfer

$$
\begin{aligned}
f_{2}\left(q^{2}\right) & =\frac{3}{2} \frac{f_{\Lambda}}{f_{\Lambda_{b}}} \frac{M^{2}}{M_{B}^{2}} \frac{1}{Q^{6}}\left(1+\frac{\lambda_{1}}{f \Lambda}\right)\left(s_{0}^{2} M_{B}^{2} e^{-\left(s_{0}-m_{\Lambda_{b}}^{2}\right) / M_{B}^{2}}+\int_{m_{b}^{2}}^{s_{0}} d s s^{2} e^{-\left(s-m_{\Lambda_{b}}^{2}\right) / M_{B}^{2}}\right) \\
f_{1}\left(q^{2}\right) & =\frac{1}{6} \frac{f_{\Lambda}}{f_{\Lambda_{b}}} \frac{M}{M_{B}^{2}} \frac{1}{Q^{6}}\left[\left(12-\frac{M^{2}}{M_{B}^{2}}\right)-3 \frac{\lambda_{1}}{f_{\Lambda}}\left(4-\frac{M^{2}}{M_{B}^{2}}\right)\right]\left(s_{0}^{3} M_{B}^{2} e^{-\left(s_{0}-m_{\Lambda_{b}}^{2}\right) / M_{B}^{2}}+\int_{m_{b}^{2}}^{s_{0}} d s s^{3} e^{-\left(s-m_{\Lambda_{b}}^{2}\right) / M_{B}^{2}}\right),
\end{aligned}
$$

from which one can observe that $f_{2}\left(q^{2}\right)$ is suppressed by $1 / Q^{2}$ compared to the expected asymptotic behavior $f_{2}\left(Q^{2}\right) \sim 1 / Q^{4}$ in terms of the analysis on the electromagnetic pion form factor [113, 114]. It is mentioned in [59] that the true asymptotic behavior of $f_{2}\left(q^{2}\right) \sim 1 / Q^{4}$ can be reproduced as a part of $O\left(\alpha_{s}^{2}\right)$ corrections, corresponding to the hard scattering mechanism, in the framework of LCSR approach. We also note that the pure contributions from the leading twist distribution amplitudes of $\Lambda$ baryon will give rise to the asymptotical behavior $f_{2}\left(Q^{2}\right) \sim 1 / Q^{8}$,

which is suppressed by one more power of $1 / Q^{2}$ compared to that from higher twist distribution amplitudes. This observation also indicates that the soft form factors of $\Lambda_{b} \rightarrow \Lambda$ are dominated by the distribution amplitudes with "wrong helicity" instead of the leading twist ones, which is in agreement with the analysis of soft contributions for nucleon form factors [59] and $\Lambda_{b} \rightarrow p l \bar{\nu}$ transition form factors [64].

\section{B. Light-cone sum rules for $\Lambda_{b} \rightarrow \Lambda+l^{+} l^{-}$}

Different from the radiative decay $\Lambda_{b} \rightarrow \Lambda \gamma$, both the matrix elements given by the tensor operator $O_{7 \gamma}$ and vector-like currents $O_{9}, O_{10}$ sandwiching between the $\Lambda_{b}$ and $\Lambda$ states can contribute to the decay $\Lambda_{b} \rightarrow \Lambda+l^{+} l^{-}$. As the former matrix elements can be directly borrowed from the radiative decay, we only need to deal with latter one within the LCSR approach. We start with the following correlation function

$$
z^{\nu} \tilde{T}_{\nu}(P, q)=i z^{\nu} \int d^{4} x e^{-i q \cdot x}\left\langle 0\left|T\left\{j_{\Lambda_{b}}(0) \tilde{j}_{\nu}(x)\right\}\right| \Lambda(P)\right\rangle,
$$

where the current $\tilde{j}_{\nu}(x)$ is given by

$$
\tilde{j}_{\nu}(x)=\bar{b}(x) \gamma_{\nu}\left(1-\gamma_{5}\right) s(x)
$$

We can write the phenomenological representation of the correlator at the hadronic level simply as

$$
z^{\nu} \tilde{T}_{\nu}=2 f_{\Lambda_{b}} \frac{\left(z \cdot P^{\prime}\right)^{2}}{m_{\Lambda_{b}}^{2}-P^{\prime 2}}\left[g_{1} \not z-g_{2} \not z \not q-G_{1} \not \gamma_{5}-G_{2} \not k \not q \gamma_{5}\right] \Lambda(P)+\ldots .
$$

where the contributions from $g_{3}$ and $G_{3}$ are proportional to the higher power of $1 / P$ in the infinite momentum kinematics $P \sim \infty, q \sim$ const, $z \sim 1 / P$ and hence are omitted in this paper. On 
the other hand, the correlation function at the quark level can be calculated in the framework of perturbative theory to the leading order of $\alpha_{s}$ as

$$
\begin{aligned}
z^{\nu} T_{\nu}=-2(z \cdot P)^{2} \not z\left(1-\gamma_{5}\right) \Lambda(P)\left\{\int \mathcal{D} x \frac{x_{3} A_{1}\left(x_{i}\right)}{\left(x_{3} P+q\right)^{2}-m_{b}^{2}}\right. \\
+M^{2} \int_{0}^{1} d x_{3} x_{3}^{2} \frac{-2 \tilde{A}_{1}\left(x_{3}\right)+\tilde{A}_{2}\left(x_{3}\right)-\tilde{A}_{3}\left(x_{3}\right)-\tilde{A}_{4}\left(x_{3}\right)+\tilde{A}_{5}\left(x_{3}\right)}{\left[\left(x_{3} P+q\right)^{2}-m_{b}^{2}\right]^{2}} \\
\left.\quad+2 M^{4} \int_{0}^{1} d x_{3} x_{3}^{3} \frac{\tilde{\tilde{A}}_{1}\left(x_{3}\right)-\tilde{\tilde{A}}_{2}\left(x_{3}\right)+\tilde{\tilde{A}}_{3}\left(x_{3}\right)+\tilde{\tilde{A}}_{4}\left(x_{3}\right)-\tilde{\tilde{A}}_{5}\left(x_{3}\right)+\tilde{\tilde{A}}_{6}\left(x_{3}\right)}{\left[\left(x_{3} P+q\right)^{2}-m_{b}^{2}\right]^{3}}\right\} \\
+2(z \cdot P)^{2} \not z \not k\left(1+\gamma_{5}\right) \Lambda(P)\left\{M \int_{0}^{1} d x_{3} x_{3} \frac{-\tilde{A}_{1}\left(x_{3}\right)+\tilde{A}_{2}\left(x_{3}\right)-\tilde{A}_{3}\left(x_{3}\right)}{\left[\left(x_{3} P+q\right)^{2}-m_{b}^{2}\right]^{2}}\right. \\
\left.+2 M^{3} \int_{0}^{1} d x_{3} x_{3}^{2} \frac{\tilde{\tilde{A}}_{1}\left(x_{3}\right)-\tilde{\tilde{A}}_{2}\left(x_{3}\right)+\tilde{\tilde{A}}_{3}\left(x_{3}\right)+\tilde{\tilde{A}}_{4}\left(x_{3}\right)-\tilde{\tilde{A}}_{5}\left(x_{3}\right)+\tilde{\tilde{A}}_{6}\left(x_{3}\right)}{\left[\left(x_{3} P+q\right)^{2}-m_{b}^{2}\right]^{3}}\right\}+\ldots .
\end{aligned}
$$

Matching the correlation function obtained in the two different representations and performing the Borel transformation with respect to the variable $P^{2}$, we can achieve the sum rules for the form factors $g_{1}$ and $g_{2}$ responsible for $\Lambda_{b} \rightarrow \Lambda+l^{+} l^{-}$decay as

$$
\begin{aligned}
f_{\Lambda_{b}} g_{1}\left(q^{2}\right) e^{-m_{\Lambda_{b}}^{2} / M_{B}^{2}}= & \int_{x_{0}}^{1} d x_{3} e^{-s / M_{B}^{2}}\left[\left(\int_{0}^{1-x_{3}} d x_{3} A_{1}\left(x_{1}, 1-x_{1}-x_{3}, x_{3}\right)\right)\right. \\
& -\frac{M^{2}}{M_{B}^{2}}\left(-2 \tilde{A}_{1}\left(x_{3}\right)+\tilde{A}_{2}\left(x_{3}\right)-\tilde{A}_{3}\left(x_{3}\right)-\tilde{A}_{4}\left(x_{3}\right)+\tilde{A}_{5}\left(x_{3}\right)\right) \\
& \left.+\frac{M^{4}}{M_{B}^{4}}\left(\tilde{\tilde{A}}_{1}\left(x_{3}\right)-\tilde{\tilde{A}}_{2}\left(x_{3}\right)+\tilde{\tilde{A}}_{3}\left(x_{3}\right)+\tilde{\tilde{A}}_{4}\left(x_{3}\right)-\tilde{\tilde{A}}_{5}\left(x_{3}\right)+\tilde{\tilde{A}}_{6}\left(x_{3}\right)\right)\right] \\
& -\frac{M^{2} x_{0}^{2} e^{-s_{0} / M_{B}^{2}}}{m_{b}^{2}-q^{2}+x_{0}^{2} M^{2}}\left[\left(-2 \tilde{A}_{1}\left(x_{0}\right)+\tilde{A}_{2}\left(x_{0}\right)-\tilde{A}_{3}\left(x_{0}\right)-\tilde{A}_{4}\left(x_{0}\right)+\tilde{A}_{5}\left(x_{0}\right)\right)\right. \\
& -\frac{M^{2}}{M_{B}^{2}}\left(\tilde{\tilde{A}}_{1}\left(x_{0}\right)-\tilde{\tilde{A}}_{2}\left(x_{0}\right)+\tilde{\tilde{A}}_{3}\left(x_{0}\right)+\tilde{\tilde{A}}_{4}\left(x_{0}\right)-\tilde{\tilde{A}}_{5}\left(x_{0}\right)+\tilde{\tilde{A}}_{6}\left(x_{0}\right)\right)(59) \\
+ & \left.M^{2} \frac{d}{d x_{0}}\left(\frac{x_{0}^{2}\left(\tilde{\tilde{A}}_{1}\left(x_{0}\right)-\tilde{\tilde{A}}_{2}\left(x_{0}\right)+\tilde{\tilde{A}}_{3}\left(x_{0}\right)+\tilde{\tilde{A}}_{4}\left(x_{0}\right)-\tilde{\tilde{A}}_{5}\left(x_{0}\right)+\tilde{\tilde{A}}_{6}\left(x_{0}\right)\right)}{m_{0}^{2}-q^{2}+x_{0}^{2} M^{2}}\right)\right],
\end{aligned}
$$

and

$$
\begin{aligned}
f_{\Lambda_{b}} g_{2}\left(q^{2}\right) e^{-m_{\Lambda_{b}}^{2} / M_{B}^{2}}= & -M\left\{\int _ { x _ { 0 } } ^ { 1 } \frac { d x _ { 3 } } { x _ { 3 } } e ^ { - s / M _ { B } ^ { 2 } } \left[\frac{1}{M_{B}^{2}}\left(-\tilde{A}_{1}\left(x_{3}\right)+\tilde{A}_{2}\left(x_{3}\right)-\tilde{A}_{3}\left(x_{3}\right)\right)\right.\right. \\
& \left.\quad-\frac{M^{2}}{M_{B}^{4}}\left(\tilde{\tilde{A}}_{1}\left(x_{3}\right)-\tilde{\tilde{A}}_{2}\left(x_{3}\right)+\tilde{\tilde{A}}_{3}\left(x_{3}\right)+\tilde{\tilde{A}}_{4}\left(x_{3}\right)-\tilde{\tilde{A}}_{5}\left(x_{3}\right)+\tilde{\tilde{A}}_{6}\left(x_{3}\right)\right)\right] \\
& +\frac{x_{0} e^{-s_{0} / M_{B}^{2}}}{m_{b}^{2}-q^{2}+x_{0}^{2} M^{2}}\left[\left(-\tilde{A}_{1}\left(x_{3}\right)+\tilde{A}_{2}\left(x_{3}\right)-\tilde{A}_{3}\left(x_{3}\right)\right)\right. \\
& \quad-\frac{M^{2}}{M_{B}^{2}}\left(\tilde{\tilde{A}}_{1}\left(x_{0}\right)-\tilde{\tilde{A}}_{2}\left(x_{0}\right)+\tilde{\tilde{A}}_{3}\left(x_{0}\right)+\tilde{\tilde{A}}_{4}\left(x_{0}\right)-\tilde{\tilde{A}}_{5}\left(x_{0}\right)+\tilde{\tilde{A}}_{6}\left(x_{0}\right)\right) \\
& \left.\left.+M^{2} x_{0} \frac{d}{d x_{0}}\left(\frac{x_{0}\left(\tilde{\tilde{A}}_{1}\left(x_{0}\right)-\tilde{\tilde{A}}_{2}\left(x_{0}\right)+\tilde{\tilde{A}}_{3}\left(x_{0}\right)+\tilde{\tilde{A}}_{4}\left(x_{0}\right)-\tilde{\tilde{A}}_{5}\left(x_{0}\right)+\tilde{\tilde{A}}_{6}\left(x_{0}\right)\right)}{m_{0}^{2}-q^{2}+x_{0}^{2} M^{2}}\right)\right]\right\} .
\end{aligned}
$$


In addition, the sum rules for form factors satisfy $G_{1}\left(q^{2}\right)=g_{1}\left(q^{2}\right), G_{2}\left(q^{2}\right)=g_{2}\left(q^{2}\right)$ to the accuracy considered in this work. These relations will be broken down with the inclusion of higher power corrections in $1 / m_{b}$ and higher order corrections in $\alpha_{s}$. Furthermore, we can also get the following relations between form factors $f_{i}$ and $g_{i}(i=1,2)$ by comparing the revelent sum rules

$$
f_{1}\left(q^{2}\right)=q^{2} g_{2}\left(q^{2}\right), \quad f_{2}\left(q^{2}\right)=g_{1}\left(q^{2}\right),
$$

which are consistent with those derived in [22, 26, 100] based on the analysis of heavy quark symmetry and the large energy symmetry.

\section{NUMERICAL ANALYSIS OF SUM RULES FOR FORM FACTORS}

Now we are going to calculate form factors $g_{2}\left(q^{2}\right)$ and $f_{2}\left(q^{2}\right)$ revelent to the $\Lambda_{b} \rightarrow \Lambda+\gamma$ and $\Lambda_{b} \rightarrow \Lambda+l^{+} l^{-}$decays numerically. Firstly, we collect the input parameters used in this paper as below [63, 115, 116, 117]

$$
\begin{array}{ll}
G_{F}=1.166 \times 10^{-2} \mathrm{GeV}^{-2}, & \left|V_{t s}\right|=41.61_{-0.80}^{+0.10} \times 10^{-3}, \\
\left|V_{t b}\right|=0.9991, & m_{b}=(4.68 \pm 0.03) \mathrm{GeV}, \\
m_{c}\left(m_{c}\right)=1.275_{-0.015}^{+0.015} \mathrm{GeV}, & m_{s}(1 \mathrm{GeV})=(142 \pm 28) \mathrm{MeV} \\
m_{\Lambda_{b}}=5.62 \mathrm{GeV}, & m_{\Lambda}=1.12 \mathrm{GeV} \\
f_{\Lambda_{b}}=3.9_{-0.2}^{+0.4} \times 10^{-3} \mathrm{GeV}^{2}, & f_{\Lambda}=6.0_{-0.4}^{+0.4} \times 10^{-3} \mathrm{GeV}^{2} \\
\lambda_{1}=-1.3_{-0.2}^{+0.2} \times 10^{-2} \mathrm{GeV}^{2}, & s_{0}=39 \pm 1 \mathrm{GeV}^{2}
\end{array}
$$

It should be noted that the normalization constants of LCDAs for $\Lambda_{b}$ and $\Lambda$ baryons, namely $f_{\Lambda_{b}}$, $f_{\Lambda}$ and $\lambda_{1}$, are all evaluated at the scale $\mu=1 \mathrm{GeV}$. As for the choice of the threshold parameter $s_{0}$, one should determine it by demanding the sum rules results to be relatively stable in allowed regions for Borel mass $M_{B}^{2}$, the value of which should be around the mass square of the corresponding first excited states.

With all the parameters, we can proceed to compute the numerical values of the form factors. In principle, the form factors $g_{2}\left(q^{2}\right)$ and $f_{2}\left(q^{2}\right)$ should not depend on the Borel masse $M_{B}^{2}$ in a complete theory. However, as we truncate the operator product expansion up to the leading conformal spin of distribution amplitudes for $\Lambda$ baryon in the leading Fock configuration and keep the perturbative expansion in $\alpha_{s}$ to leading order, a manifest dependence of the form factors on the Borel parameter $M_{B}^{2}$ would emerge in practice. Therefore, one should look for a working "window", where the results only mildly vary with respect to the Borel mass, so that the truncation is reasonable and acceptable. 
Firstly, we concentrate on the form factors at zero momentum transfer. For the form factor $f_{2}(0)$, we require that the contributions from the higher resonances and continuum states hold the fraction less than $25 \%$ in the total sum rules and the value of $f_{2}(0)$ does not vary drastically within the selected region for the Borel mass. In view of these considerations, the Borel parameter $M_{B}^{2}$ should not be too large in order to insure that the contributions from the higher states are exponentially damped as can be observed form Eqs. (51), (52), (59) and (600) and the global quark-hadron duality is satisfactory. On the other hand, the Borel mass could not be too small for the validity of OPE near the light-cone for the correlation function in deep Euclidean region, since the contributions of higher twist distribution amplitudes amount the higher order of $1 / M_{B}^{2}$ to the perturbative part. With the chosen threshold value $s_{0}=39 \mathrm{GeV}^{2}$, we indeed find the Borel platform $M_{B}^{2} \in[3.0,6.0] \mathrm{GeV}^{2}$, which is plotted in Fig. 2. The number of $f_{2}(0)$ is $0.15_{-0.02}^{+0.02}$, where we have combined the uncertainties from the variation of Borel parameters, fluctuation of threshold value, errors of $b$ quark mass and uncertainties from the non-perturbative parameters in the distribution amplitudes of $\Lambda$ baryon together. The errors on the form factor $f_{2}(0)$ are estimated within the level of $20 \%$ as expected by the general understanding of the theoretical framework. Following the same method, we can continue to estimate the numerical results for the form factor $g_{2}(0)$ within the selected Borel window as displayed in Fig. 2, The contributions from the excited resonance and continuum state are required to be less than $10 \%$ here. It can be observed that $g_{2}(0)=1.3_{-0.4}^{+0.2} \times 10^{-2} \mathrm{GeV}^{-1}$ with the given Borel window $M_{B}^{2} \in[3.0,6.0] \mathrm{GeV}^{2}$.

Next, we can further investigate the $q^{2}$ dependence of the form factors $f_{2}$ and $g_{2}$ based on the sum rules given in Eqs. (52) and (60). The OPE for the correlation function in Eqs. (41) and (55) near the light-cone is valid only at small or intermediate squared momentum transfer, $0<q^{2}<$ $m_{b}^{2}-2 m_{b} \Lambda_{Q C D}$, which ensure the good stability of the sum rules in Eqs. (51), (52), (59) and (60) with the variation of $M_{B}^{2}$. The reason is that the light-cone expansion is expected to break down when $q^{2}$ is approaching $m_{b}^{2}$ and hence contributions from the higher twist distribution amplitudes increase rapidly. In phenomenology, we extend the form factors to large squared momentum transfer by the double-pole model

$$
\xi_{i}\left(q^{2}\right)=\frac{\xi_{i}(0)}{1-a_{1} q^{2} / m_{\Lambda_{b}}^{2}+a_{2} q^{4} / m_{\Lambda_{b}}^{4}}
$$

where $\xi_{i}$ denotes the form factors $f_{2}$ and $g_{2}$. The parameters $a_{1}$ and $a_{2}$ can be fixed by the matching of form factors corresponding to the small and intermediate momentum transfer calculated in the LCSR approach. Our results are grouped in Table III, where the values of form factors in terms of the leading twist sum rules (twist-3) are also presented for a compassion. 

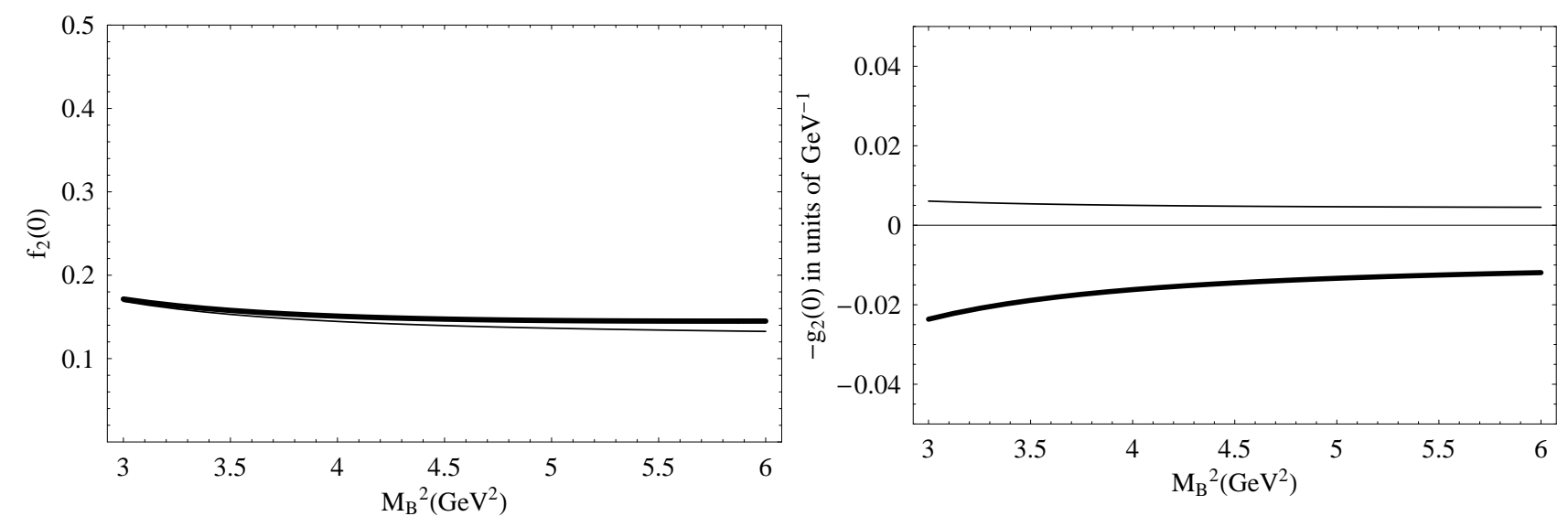

FIG. 2: Dependence of form factors $f_{2}(0)$ and $g_{2}(0)$ on the working Borel window $M_{B}^{2} \in[3.0,6.0] \mathrm{GeV}^{2}$ with the chosen threshold parameter $s_{\Lambda_{b}}^{0}=39 \mathrm{GeV}^{2}$. Here the bold line denotes the contributions from the distribution amplitudes up to twist-6, while the thin line represents the contributions from leading twist ones only.

TABLE III: Numerical results for the form factors $f_{2}(0), g_{2}(0)$ and parameters $a_{1}$ and $a_{2}$ involved in the double-pole fit of eq. (63) for both twist-3 and twist-6 sum rules with $M_{B}^{2} \in[3.0,6.0] \mathrm{GeV}^{2}, s_{0}=39 \pm 1 \mathrm{GeV}^{2}$, together with results from COZ [118] and FZOZ [119] models for distribution amplitudes of $\Lambda$ baryon in the leading twist sum rules and results obtained in QCDSR [34].

\begin{tabular}{cccccc}
\hline \hline parameter & COZ & FZOZ & QCDSR & twist-3 & up to twist-6 \\
\hline$f_{2}(0)$ & $0.74_{-0.06}^{+0.06}$ & $0.87_{-0.07}^{+0.07}$ & 0.45 & $0.14_{-0.01}^{+0.02}$ & $0.15_{-0.02}^{+0.02}$ \\
$a_{1}$ & $2.01_{-0.10}^{+0.17}$ & $2.08_{-0.09}^{+0.15}$ & 0.57 & $2.91_{-0.07}^{+0.10}$ & $2.94_{-0.06}^{+0.11}$ \\
$a_{2}$ & $1.32_{-0.08}^{+0.14}$ & $1.41_{-0.08}^{+0.11}$ & -0.18 & $2.26_{-0.08}^{+0.13}$ & $2.31_{-0.10}^{+0.14}$ \\
\hline$g_{2}(0)\left(10^{-2} \mathrm{GeV}^{-1}\right)$ & $-2.4_{-0.2}^{+0.3}$ & $-2.8_{-0.2}^{+0.4}$ & -1.4 & $-0.47_{-0.06}^{+0.06}$ & $1.3_{-0.4}^{+0.2}$ \\
$a_{1}$ & $2.76_{-0.13}^{+0.16}$ & $2.80_{-0.11}^{+0.16}$ & 2.16 & $3.40_{-0.05}^{+0.06}$ & $2.91_{-0.09}^{+0.12}$ \\
$a_{2}$ & $2.05_{-0.13}^{+0.23}$ & $2.12_{-0.13}^{+0.21}$ & 1.46 & $2.98_{-0.08}^{+0.09}$ & $2.24_{-0.13}^{+0.17}$ \\
\hline \hline
\end{tabular}

As can be observed from Table【II, the form factor $f_{2}$ is dominated by the contributions from the leading twist distribution amplitudes for $\Lambda$ baryon, and the corrections owing to the higher twist distribution amplitudes are less than $10 \%$. In contrast, the higher twist distribution amplitudes play an important role in the form factor $g_{2}$, where the numbers predicted in the twist-3 LCSR even differs from that of the whole sum rules in the sign. This observation is in agreement with the studies on semi-leptonic decay $\Lambda_{c} \rightarrow \Lambda l^{+} l$ [64] and nucleon form factor [59]. Predictions on these 
form factors can be systematically improved by including the higher conformal partial waves and higher order perturbative corrections.

Apart from the general description of distribution amplitudes based on the conformal symmetry of QCD Lagrangian, there exist two concrete models taking into account the first a few conformal partial waves for the $\Lambda$ baryons, COZ model [118], and FZOZ model [119]. The expressions of twist-3 distribution amplitudes for COZ model $A_{1}^{C O Z}\left(x_{i}\right)$ and FZOZ model $A_{1}^{F Z O Z}\left(x_{i}\right)$ can be written as

$$
\begin{aligned}
A_{1}^{C O Z}\left(x_{1}, x_{2}, x_{3}\right) & =-42 \phi_{a s}\left(x_{1}, x_{2}, x_{3}\right)\left[0.26\left(x_{3}^{2}+x_{2}^{2}\right)+0.34 x_{1}^{2}-0.56 x_{2} x_{3}-0.24 x_{1}\left(x_{2}+x_{3}\right)\right],(64) \\
A_{1}^{F Z O Z}\left(x_{1}, x_{2}, x_{3}\right) & =-42 \phi_{a s}\left(x_{1}, x_{2}, x_{3}\right)\left[0.093\left(x_{3}^{2}+x_{2}^{2}\right)+0.376 x_{1}^{2}-0.194 x_{2} x_{3}-0.207 x_{1}\left(x_{2}+x_{3}\right)\right], \\
\phi_{a s}\left(x_{1}, x_{2}, x_{3}\right) & =120 x_{1} x_{2} x_{3} .
\end{aligned}
$$

Substituting the above distribution amplitudes for $\Lambda$ baryon into sum rules Eqs. (52) and (60) and repeating the same procedure, we can get the parameters for $a_{1}$ and $a_{2}$ accounting for the form factor $f_{2}$ and $g_{2}$ as grouped in Table III. From the table, we can see that soft contributions to the transition form factors $f_{2}$ and $g_{2}$ in the $\mathrm{COZ}$ and FZOZ models are approximately five times larger than that for the LCDAs of $\Lambda$ baryon based on the conformal spin expansion. This is similar to that observed in the studies of pion form factor [113], nucleon form factor [59] and also $\Lambda_{c} \rightarrow \Lambda$ transition [64].

The form factors are also calculated in the QCDSR [34] using the heavy quark symmetry to reduce the number of independent form factors. We have translated their results with the help of Eq. (33-36). It can be observed from Table III that the numbers of $g_{2}(0)$ obtained in QCDSR differ from that extracted from twist- 6 sum rules on the light-cone in the sign. Besides, $f_{2}(0)$ in the framework of QCDSR is about three times larger than that given by LCSR approach presented here. In particular, the $q^{2}$ dependence of the from factor $f_{2}$ between these two methods are quite different. It grows slowly with the increase of squared momentum transfer $q^{2}$ in QCDSR to reach 0.83 at the maximal momentum transfer. But it rises drastically in our approach with the increase of $q^{2}$ to reach 2.3 at $q_{\max }^{2}=\left(m_{\Lambda_{b}}-m_{\Lambda}\right)^{2}=20.3 \mathrm{GeV}^{2}$. Such differences between form factors will lead to quite different predictions on values of decay width and forward-backward asymmetry for semi-leptonic decay $\Lambda_{b} \rightarrow \Lambda l^{+} l^{-}$.

The hard contributions to the form factor $f_{2}(0)$ involving two hard gluons exchange have also been investigated [35] to the leading twist distribution amplitudes for $\Lambda$ baryon in the framework of PQCD approach. The values of $f_{2}(0)$ is computed as $(1.2 \sim 1.6) \times 10^{-2}$, which is about one order smaller than the form factor presented here in terms of the LCSR approach. As a matter of 
fact, large soft corrections have been observed in the non-leptonic charmed meson decays in the perturbative QCD approach based on $k_{T}$ factorization [120]. Therefore, it seems that the form factors responsible for $\Lambda_{b} \rightarrow \Lambda$ transition may be dominated by the soft gluons exchange between valence quarks inside the $\Lambda_{b}$ and $\Lambda$ baryons.

\section{PHENOMENOLOGY OF $\Lambda_{b} \rightarrow \Lambda+\gamma$ AND $\Lambda_{b} \rightarrow \Lambda+l^{+} l^{-}$}

Utilizing the form factors derived above, we can now proceed to perform the calculations of decay rate, polarization asymmetry and forward-backward asymmetry.

\section{A. Decay width and $\Lambda$ polarization asymmetry of $\Lambda_{b} \rightarrow \Lambda+\gamma$}

In this subsection, we present the formulae of decay rate and polarization asymmetry of $\Lambda$ baryon for $\Lambda_{b} \rightarrow \Lambda+\gamma$, the latter of which can be used to analyze the helicity structures of effective Lagrangian at the quark level. The four-spin vector $s^{\mu}$ of $\Lambda$ baryon can be defined in its rest frame as

$$
\left(s^{\mu}\right)_{r . s .}=(0, \hat{\xi})
$$

which can be directly transformed into the rest frame of $\Lambda_{b}$ baryon by the Lorentz boost

$$
s^{\mu}=\left(\frac{\mathbf{p}_{\Lambda} \cdot \hat{\xi}}{m_{\Lambda}}, \hat{\xi}+\frac{s_{0}}{E_{\Lambda}+m_{\Lambda}} \mathbf{p}_{\Lambda}\right),
$$

with $\mathbf{p}_{\Lambda}$ and $E_{\Lambda}$ being the three-momentum and energy of $\Lambda$ baryon. Then, the following relations can be directly read

$$
v \cdot s=\frac{1-x^{2}}{1+x^{2}} \hat{\mathbf{p}} \cdot \mathbf{s}=\frac{1-x^{2}}{2 x} \hat{\mathbf{p}} \cdot \hat{\xi}
$$

where $x=m_{\Lambda} / m_{\Lambda_{b}}, \hat{\mathbf{p}}$ is a unite vector along the momentum of $\Lambda$ baryon and $v=p_{\Lambda_{b}} / m_{\Lambda_{b}}$ is the four-velocity vector of $\Lambda_{b}$.

Making use of Eqs. (27) and (28), the decay width of unpolarized $\Lambda_{b}$ into $\Lambda$ with a definite polarization vector $s$ can be derived as $[30,34]$

$\Gamma\left(\Lambda_{b} \rightarrow \Lambda \gamma\right)=\frac{\alpha_{e m} G_{F}^{2}}{64 m_{\Lambda_{b}}^{3} \pi^{4}}\left|V_{t b}\right|^{2}\left|V_{t s}\right|^{2}\left|C_{7}^{e f f}\right|^{2}\left(1-x^{2}\right)^{3}\left(m_{b}^{2}+m_{s}^{2}\right)\left[f_{2}(0)\right]^{2}\left[1+\frac{2 x}{1-x^{2}} \frac{m_{b}^{2}-m_{s}^{2}}{m_{b}^{2}+m_{s}^{2}}(v \cdot s)\right](69)$

For the convenience of comparing with the experimental data, we can rewrite the Eq. (69), in the

$$
\Gamma\left(\Lambda_{b} \rightarrow \Lambda \gamma\right)=\frac{1}{2} \Gamma_{0}[1+\alpha \hat{\mathbf{p}} \cdot \mathbf{s}]=\frac{1}{2} \Gamma_{0}\left[1+\alpha^{\prime} \hat{\mathbf{p}} \cdot \hat{\xi}\right]
$$


TABLE IV: Decay branching ratios (BR) of $\Lambda_{b} \rightarrow \Lambda \gamma$ calculated in the LCSR approach with distribution amplitudes of $\Lambda$ baryon in terms of the conformal spin expansion with only twist- 3 and up to twist- 6 together with results from $\mathrm{COZ}$ and FZOZ model, and results based on the form factors from pole model [30], covariant oscillator quark model [31], heavy quark effective theory [32], MIT bag model 32], non-relativistic quark model [33], QCD sum rule approach [34] and perturbative QCD approach [35], respectively.

\begin{tabular}{cccccccc}
\hline \hline Model of DAs & twist-3 & up to twist-6 & COZ & FZOZ & & \\
\hline BR $\left(\times 10^{-5}\right)$ & $0.63_{-0.12}^{+0.17}$ & $0.73_{-0.15}^{+0.15}$ & $16_{-4}^{+2}$ & $22_{-5}^{+5}$ & & & \\
\hline Model & PM & COQM & HQET & BM & NRQM & QCDSR & PQCD \\
\hline BR $\left(\times 10^{-5}\right)$ & $1.0 \sim 4.5$ & 0.23 & $0.8 \sim 1.5$ & 0.4 & 0.27 & $3.7 \pm 0.5$ & $(0.0043 \sim 0.0086)$ \\
\hline \hline
\end{tabular}

with

$$
\Gamma_{0}=\frac{\alpha_{e m} G_{F}^{2}}{32 m_{\Lambda_{b}}^{3} \pi^{4}}\left|V_{t b}\right|^{2}\left|V_{t s}\right|^{2}\left|C_{7}^{e f f}\right|^{2}\left(1-x^{2}\right)^{3}\left(m_{b}^{2}+m_{s}^{2}\right)\left[f_{2}(0)\right]^{2}
$$

and

$$
\alpha=\frac{2 x}{1+x^{2}} \alpha^{\prime}=\frac{2 x}{1+x^{2}} \frac{m_{b}^{2}-m_{s}^{2}}{m_{b}^{2}+m_{s}^{2}} .
$$

As can be observed from Eq. (172), the polarization parameters $\alpha$ and $\alpha^{\prime}$ are free of the pollution due to strong interactions [34, 83] and only depend on the relative strength of left- and right- handed couplings between quarks. Utilizing the inputs given in Eq. (62), we can get the polarization parameters as $\alpha=0.381 \pm 0.001, \alpha^{\prime}=0.998 \pm 0.001$. Any distinct deviations from these values would indicate the new physics beyond the SM.

Substituting the form factor $f_{2}(0)$ calculated in the last section into Eq. (71), we can achieve the decay rate of radiative decay $\Lambda_{b} \rightarrow \Lambda+\gamma$ as shown in the Table IV. Predictions on the branching ratio(BR) of $\Lambda_{b} \rightarrow \Lambda \gamma$ can vary even by the order of magnitude adopting different models of distribution amplitudes for $\Lambda$ baryon. We also collect the results of decay rate for $\Lambda_{b} \rightarrow \Lambda+\gamma$ computed in other approaches in Table IV, from which we can find that different methods give quite different predictions. Unfortunately, only the upper bound $1.3 \times 10^{-3}$ for $\mathrm{BR}$ of $\Lambda_{b} \rightarrow \Lambda \gamma$ decay is available in experiment at present, so we have to wait for more experimental data to discriminate existing models. 


\section{B. Decay width and dilepton distributions of $\Lambda_{b} \rightarrow \Lambda+l^{+} l^{-}$}

We adopt the transition form factors calculated in the sum rules up to the twist- 6 distribution amplitudes of $\Lambda$ baryon. The long-distance effects originated from $c \bar{c}$ resonances on the $\operatorname{BR}\left(\Lambda_{b} \rightarrow\right.$ $\Lambda l^{+} l^{-}$) are also discussed. The differential decay width of $\Lambda_{b} \rightarrow \Lambda l^{+} l^{-}$in the rest frame of $\Lambda_{b}$ baryon can be written as [115],

$$
\frac{d \Gamma\left(\Lambda_{b} \rightarrow \Lambda l^{+} l^{-}\right)}{d q^{2}}=\frac{1}{(2 \pi)^{3}} \frac{1}{32 m_{\Lambda_{b}}^{3}} \int_{u_{\min }}^{u_{\max }}\left|\widetilde{M}_{\Lambda_{b} \rightarrow \Lambda l^{+} l^{-}}\right|^{2} d u
$$

where $u=\left(p_{\Lambda}+p_{l^{-}}\right)^{2}$ and $q^{2}=\left(p_{l^{+}}+p_{l^{-}}\right)^{2} ; p_{\Lambda}, p_{l^{+}}$and $p_{l^{-}}$are the four-momenta vectors of $\Lambda$,

$l^{+}$and $l^{-}$respectively. $\widetilde{M}_{\Lambda_{b} \rightarrow \Lambda l^{+} l^{-}}$is the decay amplitude after integrating over the angle between the $l^{-}$and $\Lambda$ baryon. The upper and lower limits of $u$ are given by

$$
\begin{aligned}
& u_{\text {max }}=\left(E_{\Lambda}^{*}+E_{l}^{*}\right)^{2}-\left(\sqrt{E_{\Lambda}^{* 2}-m_{\Lambda}^{2}}-\sqrt{E_{l}^{* 2}-m_{l}^{2}}\right)^{2}, \\
& u_{\text {min }}=\left(E_{\Lambda}^{*}+E_{l}^{*}\right)^{2}-\left(\sqrt{E_{\Lambda}^{* 2}-m_{\Lambda}^{2}}+\sqrt{E_{l}^{* 2}-m_{l}^{2}}\right)^{2} ;
\end{aligned}
$$

where $E_{\Lambda}^{*}$ and $E_{l}^{*}$ are the energies of $\Lambda$ and $l^{-}$in the rest frame of lepton pair

$$
E_{\Lambda}^{*}=\frac{m_{\Lambda_{b}}^{2}-m_{\Lambda}^{2}-q^{2}}{2 \sqrt{q^{2}}}, \quad E_{l}^{*}=\frac{q^{2}}{2 \sqrt{q^{2}}} .
$$

We achieve the invariant dilepton mass distribution for $\Lambda_{b} \rightarrow \Lambda+l^{+} l^{-}(l=\mu, \tau)$ with and without long-distance contributions as plotted in Fig. 3, where the appearance of large enhancement at the end point $q^{2}=0$ for $\Lambda_{b} \rightarrow \Lambda+\mu^{+} \mu^{-}$is due to the factor $1 / q^{2}$ involved in the operator $O_{7 \gamma}$ and $h\left(z, s^{\prime}\right)$ term in Wilson coefficient $C_{9}^{e f f}$ in the effective Hamiltonian for $b \rightarrow s l^{+} l^{-}$transition. The dilepton mass distribution of $\Lambda_{b} \rightarrow \Lambda+l^{+} l^{-}(l=\mu, \tau)$ without long-distance contributions peaks at higher invariant masses in Fig. 3, which is much different from that given in [22]. The reason is that the form factors grow more drastically with the increase of squared momentum transfer $q^{2}$ in LCSR than that obtained in QCDSR, which is also found in the semi-leptonic decay $\Lambda_{b} \rightarrow p l \bar{\nu}$ [63].

Integrating Eq. (73), we get the branching fractions of $\Lambda_{b} \rightarrow \Lambda+l^{+} l^{-}(l=\mu, \tau)$ as displayed in Table V] together with the results computed in the framework of QCDSR [24] and PM [24] for a comparison. The QCDSR result [24] for decay rate of $\Lambda_{b} \rightarrow \Lambda+\tau^{+} \tau^{-}$without long-distance effects is much smaller than that presented in this work, which can be attributed to the larger transition form factors predicted in LCSR due to the sensitive dependence on the momentum transfer $q^{2}$.

We are now ready to investigate the effects of magnetic penguin operator $O_{7 \gamma}$ in the semileptonic decay $\Lambda_{b} \rightarrow \Lambda+l^{+} l^{-}(l=\mu, \tau)$. Without the operator $O_{7 \gamma}$, the $B R\left(\Lambda_{b} \rightarrow \Lambda l^{+} l^{-}\right)$is 16 


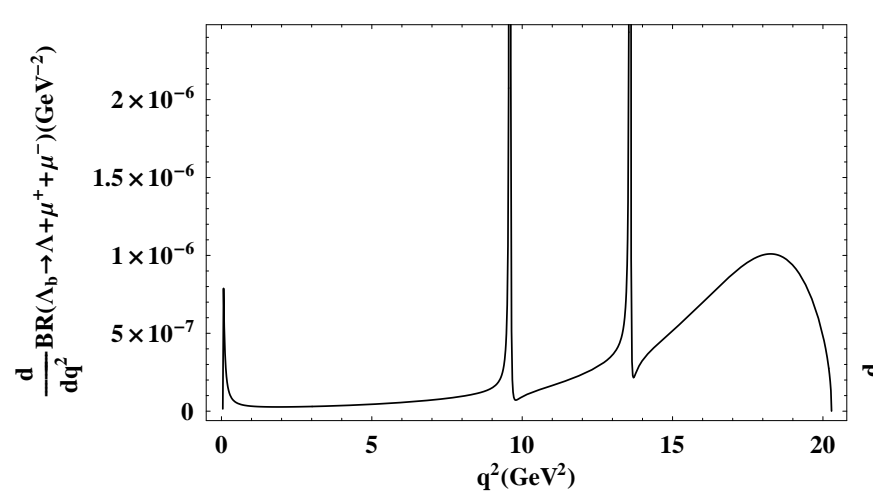

(a)

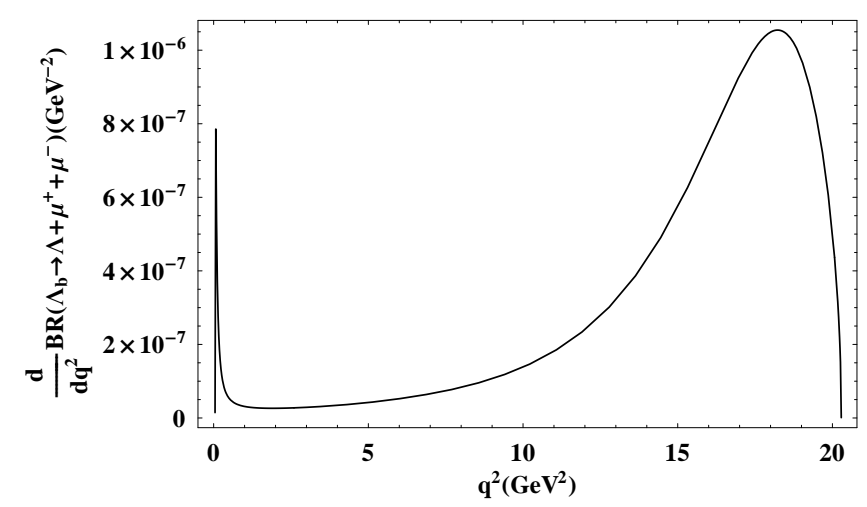

(c)

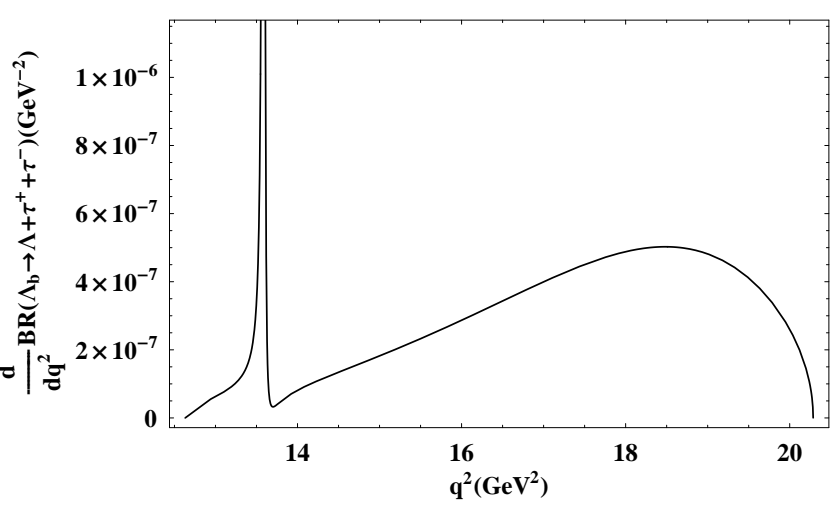

(b)

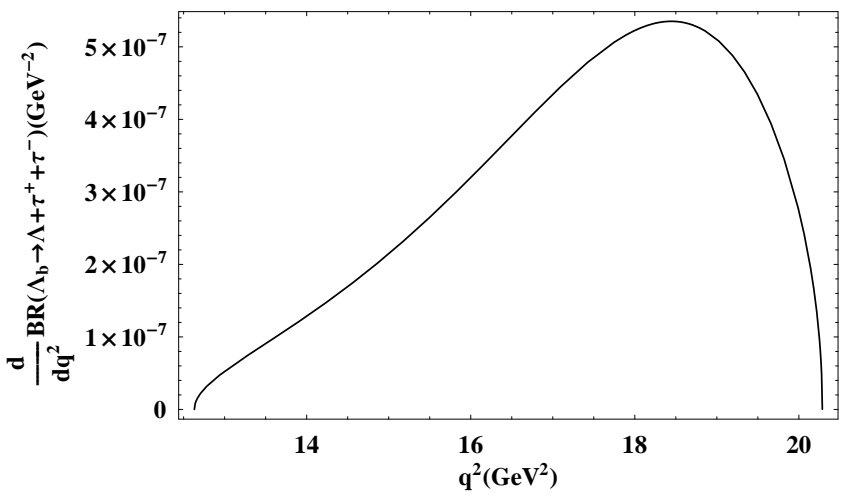

(d)

FIG. 3: The differential width for the $\Lambda_{b} \rightarrow \Lambda l^{+} l^{-}(l=\mu, \tau)$ decays as functions of $q^{2}$ with long-distance contributions $(\mathrm{a}, \mathrm{b})$ and without long-distance contributions $(\mathrm{c}, \mathrm{d})$

$\%$ and $27 \%$ smaller for final states being $\mu^{+} \mu^{-}$and $\tau^{+} \tau^{-}$, respectively. However, the branching fractions can be $38 \%$ and $57 \%$ smaller for these two modes, respectively, if we adopt the same magnitude of $C_{7}^{e f f}$ as that in the SM but with an opposite sign. This confirms the conclusion of ref. [22] that $B R\left(\Lambda_{b} \rightarrow \Lambda l^{+} l^{-}\right)$can serve as a promising quantity to explore the new physics effects as well as constrain parameter space of various models beyond the SM.

Roughly speaking, the long-distance effects on the decay rate for semi-leptonic decays of $\Lambda_{b} \rightarrow$ $\Lambda+l^{+} l^{-}$can be given by

$$
B R_{L D}\left(\Lambda_{b} \rightarrow \Lambda+l^{+} l^{-}\right)=\sum_{i} B R\left(\Lambda_{b} \rightarrow \Lambda+\Psi_{i}\right) \times B R\left(\Psi_{i} \rightarrow l^{+} l^{-}\right)
$$


TABLE V: Decay branching ratios $\left(10^{-6}\right)$ of $\Lambda_{b} \rightarrow \Lambda+l^{+} l^{-}(l=\mu, \tau)$ with and without long-distance contributions based on the form factors calculated in this work using light-cone sum rules and that from QCD sum rules and pole model

\begin{tabular}{c|cc|cc}
\hline \hline Model & \multicolumn{2}{|c|}{$\Lambda_{b} \rightarrow \Lambda+\mu^{+} \mu^{-}$} & \multicolumn{2}{c}{$\Lambda_{b} \rightarrow \Lambda+\tau^{+} \tau^{-}$} \\
& without LD with LD & without LD with LD \\
\hline LCSR (this work) & $6.1_{-1.7}^{+5.8}$ & $39_{-11}^{+23}$ & $2.1_{-0.6}^{+2.3}$ & $4.0_{-1.1}^{+3.7}$ \\
\hline QCDSR [24] & 2.1 & 53 & 0.18 & 11 \\
\hline PM [24] & 1.2 & 36 & 0.26 & 9.0 \\
\hline \hline
\end{tabular}

Utilizing the experimental data on the leptonic decays of $J / \psi$ and $\Psi(2 S)$ [115],

$$
\begin{aligned}
& B R\left(J / \Psi \rightarrow \mu^{+} \mu^{-}\right)=(5.93 \pm 0.06) \%, \quad B R\left(\Psi(2 S) \rightarrow \mu^{+} \mu^{-}\right)=(7.3 \pm 0.8) \times 10^{-3}, \\
& B R\left(\Psi(2 S) \rightarrow \tau^{+} \tau^{-}\right)=(2.8 \pm 0.7) \times 10^{-3},
\end{aligned}
$$

we can achieve the following relation

$$
\begin{aligned}
\frac{B R_{L D}\left(\Lambda_{b} \rightarrow \Lambda+\mu^{+} \mu^{-}\right)}{B R_{L D}\left(\Lambda_{b} \rightarrow \Lambda+\tau^{+} \tau^{-}\right)} & =\frac{B R\left(J / \Psi \rightarrow \mu^{+} \mu^{-}\right)+B R\left(\Psi(2 S) \rightarrow \mu^{+} \mu^{-}\right)}{B R\left(\Psi(2 S) \rightarrow \tau^{+} \tau^{-}\right)} \\
& =23.8
\end{aligned}
$$

where the assumption $B R\left(\Lambda_{b} \rightarrow \Lambda+J / \psi\right)=B R\left(\Lambda_{b} \rightarrow \Lambda+\psi(2 S)\right)$ has been used in the above derivations. We can see that this naive estimation is consistent with that computed in this work, but the number got in Ref. [24] is somewhat smaller.

We then concentrate on the role of long-distance effects played in the decay rates for both final states $\mu^{+} \mu^{-}$and $\tau^{+} \tau^{-}$within LCSR approach. As can be seen, $\Lambda_{b} \rightarrow \Lambda+\mu^{+} \mu^{-}$decay is dominated by the long-distance contributions in the vicinity of the $c \bar{c}$ resonance region, which is approximately $6 \sim 7$ times larger than that from short-distance contributions. In contrast to the case of final states with $\mu^{+} \mu^{-}$, the long-distance contributions are almost the same size as that from short-distance in $\Lambda_{b} \rightarrow \Lambda+\tau^{+} \tau^{-}$decay, which differs from that in the other two methods.

\section{C. $\Lambda$ polarization asymmetry of $\Lambda_{b} \rightarrow \Lambda+l^{+} l^{-}$}

Similar to the radiative decay $\Lambda_{b} \rightarrow \Lambda+\gamma$, we can write the differential decay width for $\Lambda_{b} \rightarrow \Lambda+l^{+} l^{-}$with respect to the squared momentum transfer $q^{2}$ in the following form

$$
\frac{d \Gamma\left(\Lambda_{b} \rightarrow \Lambda l^{+} l^{-}\right)}{d q^{2}}=A\left(q^{2}\right)+p_{\Lambda_{b}} \cdot s B\left(q^{2}\right) .
$$




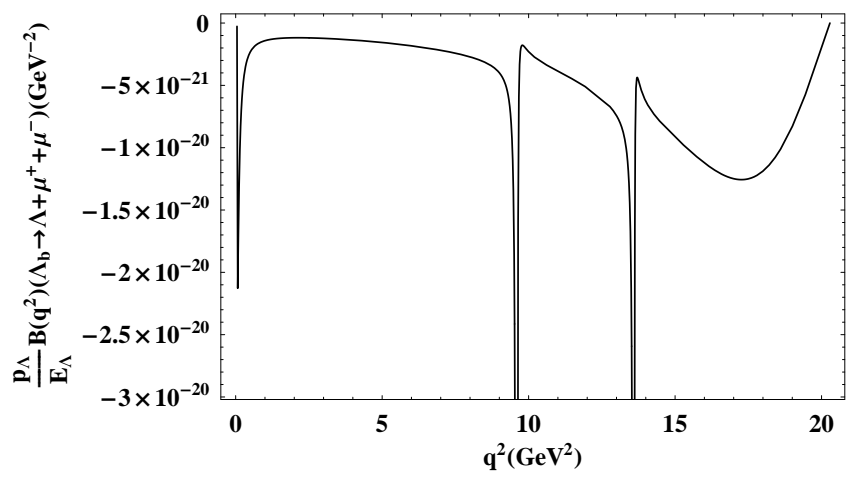

(a)

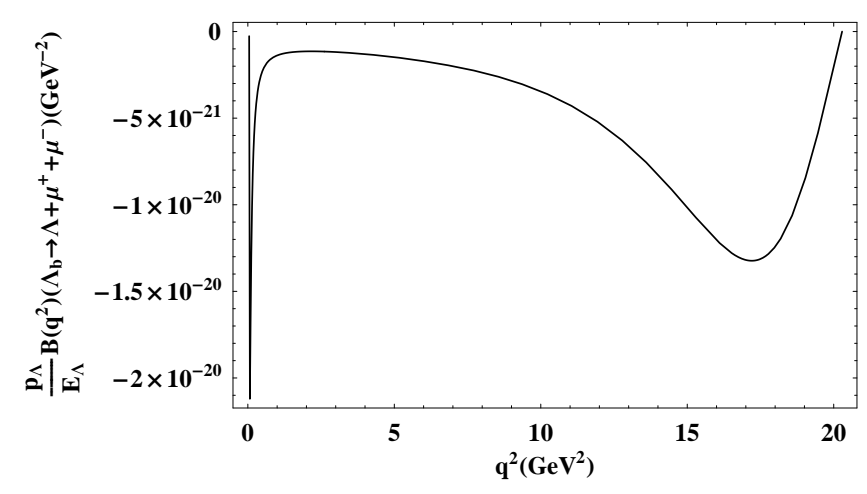

(c)

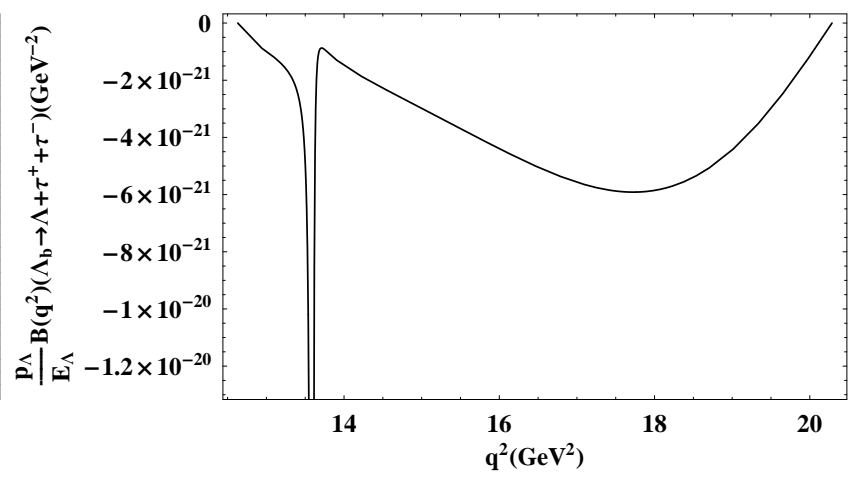

(b)

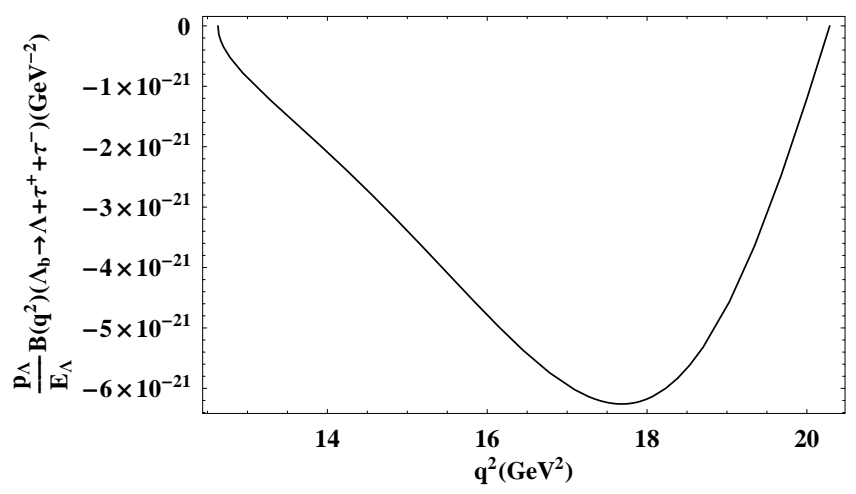

(d)

FIG. 4: Spin-dependent term $\frac{\mathbf{p}_{\Lambda}}{E_{\Lambda}} B\left(q^{2}\right)$ as a function of $q^{2}$ with long-distance contributions (a, b) and without long-distance contributions (c, d)

The $q^{2}$ dependence of the function " $\frac{\mathbf{p}_{\Lambda}}{E_{\Lambda}} B\left(q^{2}\right)$ " describing the polarization of $\Lambda$ baryon is plotted in Fig. 4 for both the cases with and without LD contributions. The function $A\left(q^{2}\right)$ is just the invariant dilepton mass distributions discussed in the previous subsection.

Integrating the differential decay width over $q^{2}$, the decay width of $\Lambda_{b} \rightarrow \Lambda+l^{+} l^{-}$with polarized $\Lambda$ baryon can be written in the form of Eq. (70)

$$
\Gamma\left(\Lambda_{b} \rightarrow \Lambda l^{+} l^{-}\right)=\frac{1}{2} \Gamma_{0}[1+\alpha \hat{\mathbf{p}} \cdot \mathbf{s}]=\frac{1}{2} \Gamma_{0}\left[1+\alpha^{\prime} \hat{\mathbf{p}} \cdot \hat{\xi}\right]
$$

where $\Gamma_{0}$ is the total decay width of $\Lambda_{b} \rightarrow \Lambda+l^{+} l^{-}$

$$
\Gamma_{0}=2 \int_{q_{\min }^{2}}^{q_{\max }^{2}} A\left(q^{2}\right) d q^{2},
$$


TABLE VI: Polarization asymmetry parameter $\alpha$ and $\alpha^{\prime}$ of $\Lambda$ baryon with and without long-distance contributions based on the form factors calculated in light-cone sum rules

\begin{tabular}{r|rr|rr}
\hline \hline & \multicolumn{2}{|c|}{$\Lambda_{b} \rightarrow \Lambda+\mu^{+} \mu^{-}$} & \multicolumn{2}{c}{$\Lambda_{b} \rightarrow \Lambda+\tau^{+} \tau^{-}$} \\
& without LD & with LD & without LD & with LD \\
\hline$\alpha$ & $-0.36_{-0.02}^{+0.05}$ & $-0.50_{-0.01}^{+0.04}$ & $-0.28_{-0.03}^{+0.03}$ & $-0.27_{-0.03}^{+0.03}$ \\
$\alpha^{\prime}$ & $-0.55_{-0.04}^{+0.09}$ & $-0.88_{-0.03}^{+0.07}$ & $-0.36_{-0.03}^{+0.04}$ & $-0.39_{-0.03}^{+0.06}$ \\
\hline \hline
\end{tabular}

with $q_{\text {min }}^{2}=4 m_{l}^{2}$ and $q_{\text {max }}^{2}=\left(m_{\Lambda_{b}}-m_{\Lambda}\right)^{2}$. Besides, $\alpha$ and $\alpha^{\prime}$ are the polarization asymmetry parameters, whose manifest expressions can be expressed in terms of function $B\left(q^{2}\right)$ as

$$
\alpha=\frac{2 m_{\Lambda_{b}}}{\Gamma_{0}} \int_{q_{\min }^{2}}^{q_{\max }^{2}} \frac{\left|\mathbf{p}_{\Lambda}\right|}{E_{\Lambda}} B\left(q^{2}\right) d q^{2}, \quad \alpha^{\prime}=\frac{2 m_{\Lambda_{b}}}{\Gamma_{0}} \int_{q_{\min }^{2}}^{q_{\max }^{2}} \frac{\left|\mathbf{p}_{\Lambda}\right|}{m_{\Lambda}} B\left(q^{2}\right) d q^{2},
$$

with $\left|\mathbf{p}_{\Lambda}\right|=\sqrt{\left(\frac{m_{\Lambda_{b}}^{2}+m_{\Lambda}^{2}-q^{2}}{2 m_{\Lambda_{b}}}\right)^{2}-m_{\Lambda}^{2}}$ being the magnitude of three-momentum for $\Lambda$ baryon in the rest frame of $\Lambda_{b}$. The numerical results of polarization variables $\alpha$ and $\alpha^{\prime}$ are grouped in Table VI, As can be seen, the long-distance contributions have small effects on the polarization asymmetry for decay of $\Lambda_{b} \rightarrow \Lambda+\tau^{+} \tau^{-}$, however, they can modify the polarization asymmetry for the mode $\Lambda_{b} \rightarrow \Lambda+\mu^{+} \mu^{-}$remarkably. The reason is that only $c \bar{c}$ resonances above (including) the $\Psi(2 S)$ threshold can have influences on the long-distance contributions for $\Lambda_{b} \rightarrow \Lambda+\tau^{+} \tau^{-}$, which are much suppressed compared with that from $J / \psi$ in the decay of $\Lambda_{b} \rightarrow \Lambda+\mu^{+} \mu^{-}$. The value of $\alpha$ corresponding to the channel $\Lambda_{b} \rightarrow \Lambda+\mu^{+} \mu^{-}$is consistent with $-0.54_{-0.04}^{+0.04}$ that calculated in [34] within the error bar.

\section{Forward-backward asymmetry of $\Lambda_{b} \rightarrow \Lambda+l^{+} l^{-}$}

For the illustration of forward-backward asymmetry, we consider the following double patrial differential decay rates for the decays of $\Lambda_{b} \rightarrow \Lambda l^{+} l^{-}$

$$
\frac{d^{2} \Gamma\left(q^{2}, z\right)}{d q^{2} d z}=\frac{1}{(2 \pi)^{3}} \frac{1}{64 m_{\Lambda_{b}}^{3}} \lambda^{1 / 2}\left(m_{\Lambda_{b}}^{2}, m_{\Lambda}^{2}, q^{2}\right) \sqrt{1-\frac{4 m_{l}^{2}}{q^{2}}}\left|\widetilde{M}_{\Lambda_{b} \rightarrow \Lambda l^{+} l^{-}}\right|^{2},
$$

where $z=\cos \theta$ and $\theta$ is the angle between the momentum of $\Lambda_{b}$ baryon and $l^{-}$in the dilepton rest frame; $\lambda(a, b, c)=a^{2}+b^{2}+c^{2}-2 a b-2 a c-2 b c$. Following Refs. [22, 91], the differential and normalized forward-backward asymmetries for the semi-leptonic decay $\Lambda_{b} \rightarrow \Lambda l^{+} l^{-}$can be defined as

$$
\frac{d A_{F B}\left(q^{2}\right)}{d q^{2}}=\int_{0}^{1} d z \frac{d^{2} \Gamma\left(q^{2}, z\right)}{d q^{2} d z}-\int_{-1}^{0} d z \frac{d^{2} \Gamma\left(q^{2}, z\right)}{d q^{2} d z} .
$$


and

$$
A_{F B}\left(q^{2}\right)=\frac{\int_{0}^{1} d z \frac{d^{2} \Gamma\left(q^{2}, z\right)}{d q^{2} d z}-\int_{-1}^{0} d z \frac{d^{2} \Gamma\left(q^{2}, z\right)}{d q^{2} d z}}{\int_{0}^{1} d z \frac{d^{2} \Gamma\left(q^{2}, z\right)}{d q^{2} d z}+\int_{-1}^{0} d z \frac{d^{2} \Gamma\left(q^{2}, z\right)}{d q^{2} d z}} .
$$

Making use of the decay amplitude in Eq. (2), the differential forward-backward asymmetry for decays of $\Lambda_{b} \rightarrow \Lambda+l^{+} l^{-}$can be calculated as

$$
\frac{d A_{F B}\left(q^{2}\right)}{d q^{2}}=\frac{G_{F}^{2} \alpha_{e m}^{2}\left|V_{t b} V_{t s}^{*}\right|^{2}}{256 m_{\Lambda_{b}}^{3} \pi^{5}} \lambda\left(m_{\Lambda_{b}^{2}}, m_{\Lambda}^{2}, q^{2}\right)\left(1-\frac{4 m_{l}^{2}}{q^{2}}\right) R_{F B}\left(q^{2}\right)
$$

with

$$
\begin{aligned}
R_{F B}\left(q^{2}\right)= & 2\left[\left(m_{s} m_{\Lambda}+m_{b} m_{\Lambda_{b}}\right) f_{2}^{2}-m_{s}\left(m_{\Lambda}^{2}-m_{\Lambda_{b}}^{2}+q^{2}\right) f_{2} g_{2}+\left(m_{s} m_{\Lambda}-m_{b} m_{\Lambda_{b}}\right) q^{2} g_{2}^{2}\right] \operatorname{Re}\left(C_{7}^{e f f} C_{10}^{*}\right) \\
& +\left[\left(f_{2}-g_{2} m_{\Lambda}\right)^{2}-g_{2}^{2} m_{\Lambda_{b}}^{2}\right] q^{2} \operatorname{Re}\left(C_{9}^{e f f} C_{10}^{*}\right)
\end{aligned}
$$

where we have retained masses for both the lepton and strange quark. In the limit of $m_{s} \rightarrow 0$, our results will be the same as that in Ref. [22]. The differential forward-backward asymmetry for semi-leptonic decay of $\Lambda_{b} \rightarrow \Lambda l^{+} l^{-}$only depends on the following two combinations of Wilson coefficients $\operatorname{Re}\left(C_{7}^{e f f} C_{10}^{*}\right)$ and $\operatorname{Re}\left(C_{9}^{e f f} C_{10}^{*}\right)$, since only terms involving $\operatorname{Tr}\left[L_{\mu}^{V} L_{\nu}^{A}\right]$ in the differential decay width can give rise to one power of " $\cos \theta$ " [22], where $L_{\mu}^{V}$ and $L_{\nu}^{A}$ represent the vector and axial-vector currents of leptonic sector. The zero position $t_{0}$ of forward-backward asymmetry is determined by the relation

$$
\operatorname{Re}\left(C_{9}^{e f f} C_{10}^{*}\right)=-\frac{2 m_{b} m_{\Lambda_{b}}}{t_{0}} \frac{f_{2}^{2}-t_{0} g_{2}^{2}}{\left(f_{2}-m_{\Lambda} g_{2}\right)^{2}-m_{\Lambda_{b}}^{2} g_{2}^{2}} \operatorname{Re}\left(C_{7}^{\text {eff }} C_{10}^{*}\right),
$$

where the small number of strange quark mass is neglected. It is easy to observe that $t_{0}$ only rely on the Wilson coefficient as well as the ratio of form factors. Utilizing the form factors computed in this work, we can derive the zero position of forward-backward asymmetry at $t_{0}=6.0 \mathrm{GeV}^{2}$ without long-distance contributions, which indicates that the forward-backward asymmetry for $\Lambda_{b} \rightarrow \Lambda+\tau^{+} \tau^{-}$could not be zero apart from the resonance regions and end points. However, the number of $t_{0}$ can shift to $3.5 \mathrm{GeV}^{2}$ using the form factors calculated in QCDSR [34], due to the quite different predictions on the ratio of from factors in these two approaches. It is mentioned in [22, 100] that the zero-position of forward-backward asymmetry is not sensitive to the form factors in the large energy limit. However, power corrections contributed by the higher twist distribution amplitudes of $\Lambda$ baryon can bring about significant effects on the ratio of form factors, which can even overwhelm the sign of form factor breaking the heavy quark symmetry. The position of the zero of the forward-backward can shift observably for the ratio of form factors with different sign. 
The distribution of normalized forward-backward asymmetry as a function of $q^{2}$ with and without long-distance contributions is presented in Fig. 5 and Fig. 6

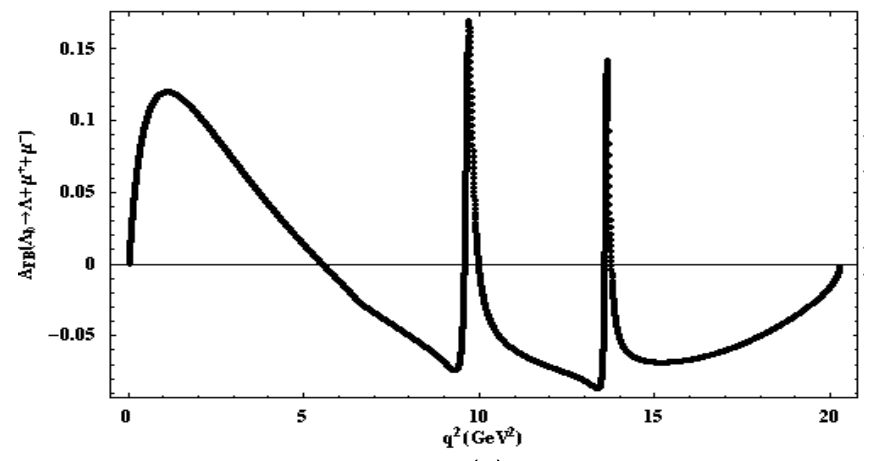

(a)

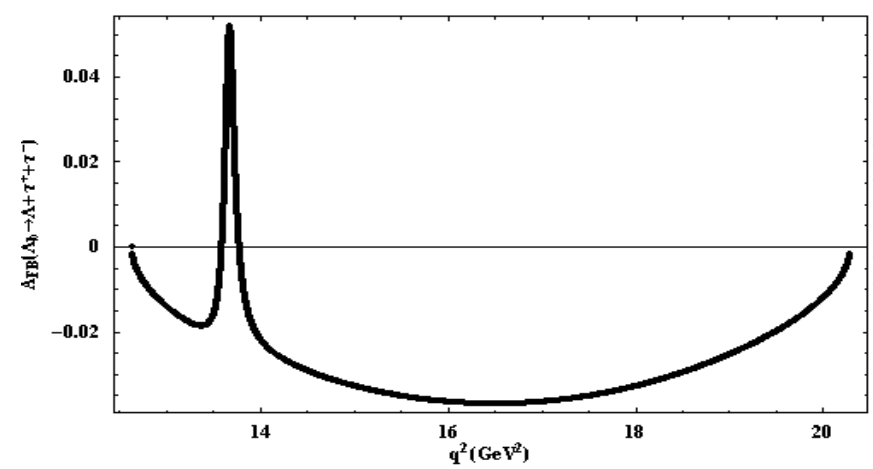

(b)

FIG. 5: Normalized forward-backward asymmetry $A_{F B}\left(q^{2}\right)$ as a function of $q^{2}$ with long-distance contributions: (a) for muon, and (b) for tauon.

It is also useful to introduce the integrated forward-backward asymmetry $\left\langle A_{F B}\right\rangle$ in order to 


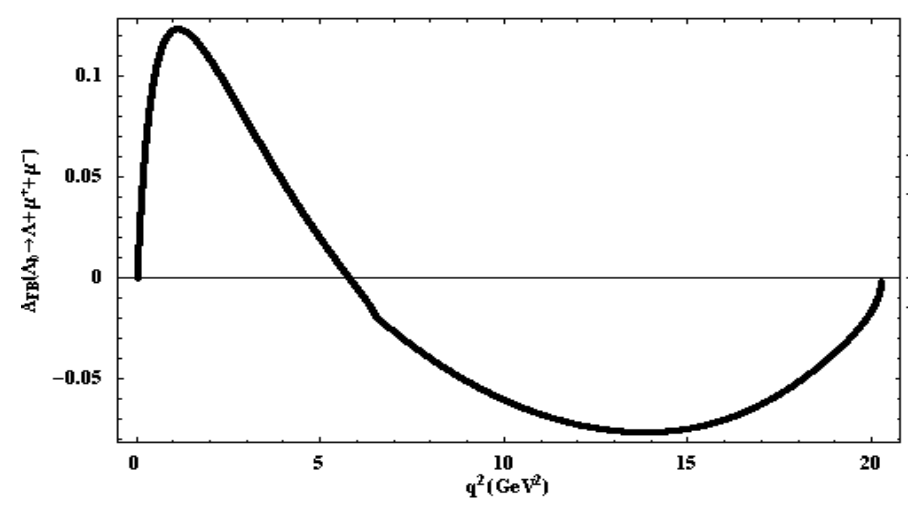

(a)

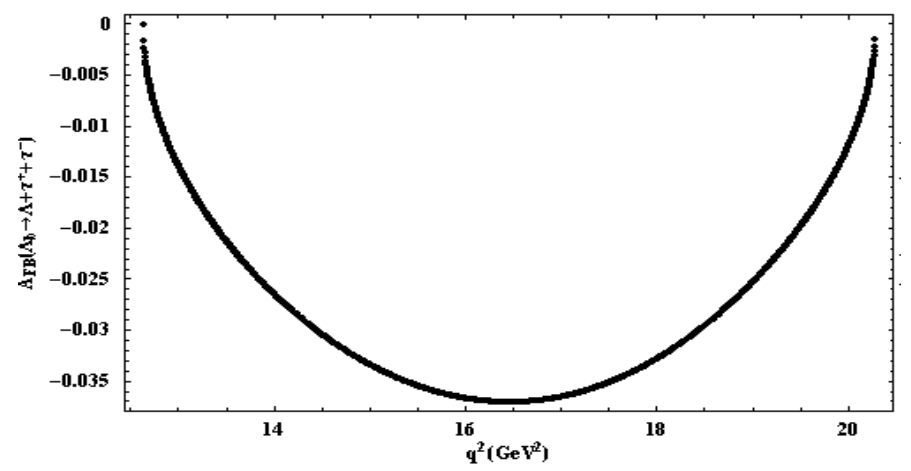

(b)

FIG. 6: Normalized forward-backward asymmetry $A_{F B}\left(q^{2}\right)$ as a function of $q^{2}$ without long-distance contributions: (a) for muon, and (b) for tauon. 
characterize the typical value of forward-backward asymmetry [22]

$$
\left\langle A_{F B}\right\rangle=\int_{t_{\min }}^{t_{\max }} A_{F B}(t) d t
$$

with $t=q^{2} / m_{\Lambda_{b}}^{2}, t_{\min }=4 m_{l}^{2} / m_{\Lambda_{b}}^{2}$ and $t_{\max }=\left(m_{\Lambda_{b}}^{2}-m_{\Lambda}^{2}\right) / m_{\Lambda_{b}}^{2}$. The numerical results of integrated forward-backward asymmetry are grouped in Table VII, where the evaluations in QCDSR are also given for a comparison. As can be observed, the predictions on the $\left\langle A_{F B}\right\rangle$ in LCSR are typically one order smaller than that in QCDSR due to the quite different predictions on the ratio of form factors. The large discrepancy may be recoiled by including the higher conformal spin contributions in the sum rules of form factors on the light-cone as well as radiative corrections for both two types of sum rules.

TABLE VII: Averaged forward-backward asymmetry (\%) for $\Lambda_{b} \rightarrow \Lambda+l^{+} l^{-}$with and without long-distance contributions based on the form factors calculated in this work using light-cone sum rules and that from QCD sum rules

\begin{tabular}{c|ccc}
\hline \hline model & \multicolumn{3}{|c}{$\Lambda_{b} \rightarrow \Lambda+\mu^{+} \mu^{-} \Lambda_{b} \rightarrow \Lambda+\tau^{+} \tau^{-}$} \\
\hline LCSR & without LD & $-1.22_{-0.73}^{+1.42}$ & $-0.67_{-0.21}^{+0.23}$ \\
(this work) & with LD & $-0.99_{-0.68}^{+1.32}$ & $-0.62_{-0.21}^{+0.22}$ \\
\hline QCDSR [22] & without LD & $-14.38_{-0.0}^{+1.14}$ & $-3.99_{-0.01}^{+0.01}$ \\
\hline \hline
\end{tabular}

\section{SUMMARY}

The study on rare decay of $\Lambda_{b} \rightarrow \Lambda+\gamma$ and $\Lambda_{b} \rightarrow \Lambda+l^{+} l^{-}$can serve as the baryonic counterparts of analysis on $B \rightarrow K^{*} \gamma[11,12,13,14,15,16,17,18,19,20,21], B \rightarrow K^{*} l^{+} l^{-}[5$, , 6, 7,, 8, 19, 10] and could also be the extension of investigations on heavy baryon decays $\Lambda_{b} \rightarrow p l \bar{\nu}$ [63] and $\Lambda_{c} \rightarrow \Lambda l \bar{\nu}$ 64]. Such decays play the role as a corner stone [121] to explore the quark-flavor structure of the $\mathrm{SM}$ as well as determine its fundamental parameters such as the CKM matrix.

Although we have achieved inspiring progress in the heavy meson decays with the help of heavy quark expansions and factorization techniques, serious and systemical studies on heavy baryon decays are comparatively behind on account of the complexity of inner structures for baryon systems. In particular, the predictions on the physical observables associating with heavy baryon decays can vary even by the orders of magnitude employing different theoretical tools. In this work, we explore the soft contributions to the form factors responsible for the $\Lambda_{b} \rightarrow \Lambda$ transition 
in terms of LCSR approach to understand the tremendous discrepancy on predictions of decay rate for $\Lambda_{b} \rightarrow \Lambda+\gamma$ between theoretical methods. More importantly, power corrections from the higher twist distribution amplitudes of $\Lambda$ baryon to the transition form factors are also investigated to the leading conformal spin in detail. It is observed that the higher twist LCDAs almost have no influences on the transition form factors reserving the heavy quark spin symmetry, while such corrections can result in significant impacts on the from factors breaking the heavy quark spin symmetry. In addition, we also find that various models for the distribution amplitudes of $\Lambda$ baryon can lead to quite different predictions on the transition form factors, which will be tested by the experiments on Tevatron and LHC in the future.

We confirm the conclusion of Ref. [34, 83] that the $\Lambda$ polarization asymmetry of $\Lambda_{b} \rightarrow \Lambda \gamma$ only relies on the relative strength of left- and right- handed couplings between quarks and is free of the pollution from the strong interaction. We also discuss some interesting observables in phenomenology, such as decay rate, dilepton distribution, polarization asymmetry and forwardbackward asymmetry of $\Lambda_{b} \rightarrow \Lambda l^{+} l^{-}$. We use the pole model to extrapolate the results of form factors calculated in LCSR to the whole physical region in view of the failure of light-cone expansion for correlation function in the large momentum transfer region. Our results indicate that the decay rate for $\Lambda_{b} \rightarrow \Lambda \mu^{+} \mu^{-}$is about five times larger than that for $\Lambda_{b} \rightarrow \Lambda \gamma$ due to the long-distance contributions from the charmonium resonance region. The polarization asymmetry of $\Lambda_{b} \rightarrow \Lambda \mu^{+} \mu^{-}$ is much sensitive to the long-distance contributions than that of $\Lambda_{b} \rightarrow \Lambda \tau^{+} \tau^{-}$, since leading resonance $J / \psi$ does not contribute to the case of final state with tauon pair. As for the integrated forward-backward asymmetry, our results for the magnitude of both muon and tauon cases are typically one order smaller than that given in QCDSR due to the quite different predictions on the ratio of transition form factors, which can also shift the zero position of forward-backward asymmetry from $t_{0}=6.0 \mathrm{GeV}^{2}$ in LCSR to $3.5 \mathrm{GeV}^{2}$ predicted by QCDSR approach.

\section{Acknowledgements}

This work is partly supported by National Science Foundation of China under Grant No. 10735080 and 10625525. The authors would like to thank T.M. Aliev, C.H. Chen, H.Y. Cheng, X.G. He, G. Hiller, C.S. Huang, M.Q. Huang, T. Huang, M. Jamil, A. Lenz and Y.L. Liu for helpful discussions. 


\section{APPENDIX A: QCD SUM RULES FOR $f_{\Lambda}, f_{\Lambda_{b}}$ AND $\lambda_{1}$}

The sum rules of $f_{\Lambda}$ and $\lambda_{1}$ have been derived in [64], we would like to collect them in this appendix for the completeness of the paper.

$$
\begin{aligned}
(4 \pi)^{4} f_{\Lambda}^{2} e^{-M^{2} / M_{B}^{2}}=\frac{2}{5} \int_{m_{s}^{2}}^{s_{\Lambda}^{0}} s(1-x)^{5} e^{-s / M_{B}^{2}} d s-\frac{b}{3} \int_{m_{s}^{2}}^{s_{\Lambda}^{0}} x(1-x)(1-2 x) e^{-s / M_{B}^{2}} \frac{d s}{s}, \\
4(2 \pi)^{4} \lambda_{1}^{2} M^{2} e^{-M^{2} / M_{B}^{2}}=\frac{1}{2} \int_{m_{s}^{2}}^{s_{0}} s^{2}\left[\left(1-x^{2}\right)\left(1-8 x+x^{2}\right)-12 x^{2} \ln x\right] e^{-s / M_{B}^{2}} d s \\
+\frac{b}{4} \int_{m_{s}^{2}}^{s_{0}}(1-x)^{2} e^{-s / M_{B}^{2}} d s-\frac{4}{3} a^{2} e^{-M^{2} / M_{B}^{2}}
\end{aligned}
$$

where $x=m_{s}^{2} / s$ and $m_{s}$ is the mass of strange quark. The values of the non-perturbative condensate at scale $\mu=1 \mathrm{GeV}$ are given by

$$
\begin{aligned}
& a=-(2 \pi)^{2}\langle\bar{q} q\rangle=0.55 \mathrm{GeV}^{3} \\
& b=(2 \pi)^{2}\left\langle\frac{\alpha_{s}}{\pi} G^{2}\right\rangle=0.47 \mathrm{GeV}^{4} .
\end{aligned}
$$

The sum rules for $f_{\Lambda_{b}}$ can be read from Eq. (A1), only with the following replacement:

$$
f_{\Lambda} \rightarrow f_{\Lambda_{b}}, m_{s} \rightarrow m_{b}, \quad s_{\Lambda}^{0} \rightarrow s_{\Lambda_{b}}^{0}
$$

With the threshold value $s_{\Lambda}^{0}=1.6 \mathrm{GeV}^{2}$, we can arrive at the numbers of $f_{\Lambda}$ and $\lambda_{1}$ as

$$
f_{\Lambda}=6.0_{-0.4}^{+0.4} \times 10^{-3} \mathrm{GeV}^{2}, \quad \lambda_{1}=-1.3_{-0.2}^{+0.2} \times 10^{-2} \mathrm{GeV}^{2},
$$

within the Borel window $M_{B}^{2} \in[1.0,2.0] \mathrm{GeV}^{2}$. In the same way, the result of $f_{\Lambda_{b}}$ can be derived as $f_{\Lambda_{b}}=3.9_{-0.2}^{+0.4} \times 10^{-3} \mathrm{GeV}^{2}$ with the selected threshold parameter $s_{\Lambda_{b}}^{0}=39 \pm 1 \mathrm{GeV}^{2}$ and Borel $\operatorname{mass} M_{B}^{2} \in[2.0,3.5] \mathrm{GeV}^{2}$.

[1] S. L. Glashow, J. Iliopoulos and L. Maiani, Phys. Rev. D 2 (1970) 1285.

[2] N. Cabibbo, Phys. Rev. Lett. 10 (1963) 531.

[3] M. Kobayashi and T. Maskawa, Prog. Theor. Phys. 49 (1973) 652.

[4] For a recent review, see A. Ali, Invited talk at the International Symposium on Contemporary Physics, NCP, Islamabad, Pakistan (March 26-30, 2007), arXiv:0712.1022 [hep-ph].

[5] T. M. Aliev, A. Ozpineci and M. Savci, Phys. Rev. D 56 (1997) 4260 arXiv:hep-ph/9612480.

[6] P. Ball and V. M. Braun, Phys. Rev. D 58 (1998) 094016 arXiv:hep-ph/9805422.

[7] T. M. Aliev, C. S. Kim and Y. G. Kim, Phys. Rev. D 62 (2000) 014026 arXiv:hep-ph/9910501. 
[8] T. M. Aliev, D. A. Demir and M. Savci, Phys. Rev. D 62 (2000) 074016 arXiv:hep-ph/9912525.

[9] W. Jaus and D. Wyler, Phys. Rev. D 41 (1990) 3405.

[10] A. Ali, P. Ball, L. T. Handoko and G. Hiller, Phys. Rev. D 61 (2000) 074024 arXiv:hep-ph/9910221.

[11] A. Ali, V. M. Braun and H. Simma, Z. Phys. C 63 (1994) 437 arXiv:hep-ph/9401277.

[12] N. G. Deshpande, P. Lo, J. Trampetic, G. Eilam and P. Singer, Phys. Rev. Lett. 59 (1987) 183.

[13] N. G. Deshpande, J. Trampetic and K. Panose, Phys. Lett. B 214 (1988) 467.

[14] M. Beneke, T. Feldmann and D. Seidel, Nucl. Phys. B 612 (2001) 25 arXiv:hep-ph/0106067.

[15] S. W. Bosch and G. Buchalla, Nucl. Phys. B 621 (2002) 459 arXiv:hep-ph/0106081.

[16] H. n. Li and G. L. Lin, Phys. Rev. D 60 (1999) 054001 arXiv:hep-ph/9812508.

[17] J. P. Lee, Phys. Rev. D 74 (2006) 074001 arXiv:hep-ph/0608087.

[18] M. Jamil Aslam and Riazuddin, Phys. Rev. D 72 (2005) 094019 arXiv:hep-ph/0509082.

[19] M. J. Aslam, Eur. Phys. J. C 49 (2007) 651 arXiv:hep-ph/0604025.

[20] M. Jamil Aslam and Riazuddin, Phys. Rev. D 75 (2007) 034004 arXiv:hep-ph/0607114.

[21] W. Wang, R. H. Li and C. D. Lü, arXiv:0711.0432 [hep-ph].

[22] C. H. Chen and C. Q. Geng, Phys. Rev. D 64 (2001) 074001 arXiv:hep-ph/0106193.

[23] C. H. Chen and C. Q. Geng, Phys. Rev. D 63 (2001) 054005 arXiv:hep-ph/0012003.

[24] C. H. Chen and C. Q. Geng, Phys. Rev. D 63 (2001) 114024 arXiv:hep-ph/0101171.

[25] C. H. Chen and C. Q. Geng, Phys. Lett. B 516 (2001) 327 arXiv:hep-ph/0101201.

[26] T. M. Aliev, A. Ozpineci and M. Savci, Nucl. Phys. B 649 (2003) 168 arXiv:hep-ph/0202120.

[27] T. M. Aliev, A. Ozpineci and M. Savci, Phys. Rev. D 67 (2003) 035007 arXiv:hep-ph/0211447.

[28] T. M. Aliev, V. Bashiry and M. Savci, Nucl. Phys. B 709 (2005) 115 arXiv:hep-ph/0407217.

[29] T. M. Aliev and M. Savci, JHEP 0605 (2006) 001 arXiv:hep-ph/0507324.

[30] T. Mannel and S. Recksiegel, J. Phys. G 24 (1998) 979 arXiv:hep-ph/9701399.

[31] R. Mohanta, A. K. Giri, M. P. Khanna, M. Ishida and S. Ishida, Prog. Theor. Phys. 102 (1999) 645 arXiv:hep-ph/9908291.

[32] H. Y. Cheng, C. Y. Cheung, G. L. Lin, Y. C. Lin, T. M. Yan and H. L. Yu, Phys. Rev. D 51 (1995) 1199 arXiv:hep-ph/9407303.

[33] H. Y. Cheng and B. Tseng, Phys. Rev. D 53, 1457 (1996) [Erratum-ibid. D 55, 1697 (1997)] arXiv:hep-ph/9502391.

[34] C. S. Huang and H. G. Yan, Phys. Rev. D 59 (1999) 114022 [Erratum-ibid. D 61 (2000) 039901] arXiv:hep-ph/9811303.

[35] X. G. He, T. Li, X. Q. Li and Y. M. Wang, Phys. Rev. D 74 (2006) 034026 arXiv:hep-ph/0606025.

[36] I. I. Balitsky, V. M. Braun and A. V. Kolesnichenko, Nucl. Phys. B 312 (1989) 509.

[37] I. I. Balitsky, V. M. Braun and A. V. Kolesnichenko, Sov. J. Nucl. Phys. 44 (1986) 1028 [Yad. Fiz. 44 (1986) 1582].

[38] V. M. Braun and I. E. Filyanov, Z. Phys. C 44 (1989) 157 [Sov. J. Nucl. Phys. 50 (1989 YAFIA,50,818830.1989) 511.1989 YAFIA,50,818]. 
[39] V. L. Chernyak and I. R. Zhitnitsky, Nucl. Phys. B 345 (1990) 137.

[40] P. Ball, V. M. Braun and H. G. Dosch, Phys. Rev. D 44 (1991) 3567.

[41] M. A. Shifman, A. I. Vainshtein and V. I. Zakharov, Nucl. Phys. B 147 (1979) 385.

[42] M. A. Shifman, A. I. Vainshtein and V. I. Zakharov, Nucl. Phys. B 147 (1979) 519.

[43] M. A. Shifman, A. I. Vainshtein and V. I. Zakharov, Nucl. Phys. B 147 (1979) 448.

[44] G. P. Lepage and S. J. Brodsky, Phys. Lett. B 87 (1979) 359.

[45] G. P. Lepage and S. J. Brodsky, Phys. Rev. D 22 (1980) 2157.

[46] S. J. Brodsky and G. P. Lepage, Phys. Rev. D 24 (1981) 1808.

[47] A. V. Efremov and A. V. Radyushkin, Phys. Lett. B 94 (1980) 245.

[48] A. V. Efremov and A. V. Radyushkin, Theor. Math. Phys. 42 (1980) 97 [Teor. Mat. Fiz. 42 (1980) $147]$.

[49] V. L. Chernyak and A. R. Zhitnitsky, JETP Lett. 25 (1977) 510 [Pisma Zh. Eksp. Teor. Fiz. 25 (1977) $544]$.

[50] V. L. Chernyak and A. R. Zhitnitsky, Sov. J. Nucl. Phys. 31 (1980) 544 [Yad. Fiz. 31 (1980) 1053].

[51] V. L. Chernyak and A. R. Zhitnitsky, Phys. Rept. 112 (1984) 173.

[52] V. M. Braun, Plenary talk given at the IVth International Workshop on Progress in Heavy Quark Physics Rostock, Germany, 20-22 September 1997 arXiv:hep-ph/9801222.

[53] P. Ball and V. M. Braun, Phys. Rev. D 55 (1997) 5561 arXiv:hep-ph/9701238.

[54] P. Ball, JHEP 9809 (1998) 005 arXiv:hep-ph/9802394.

[55] A. Khodjamirian, R. Ruckl, S. Weinzierl, C. W. Winhart and O. I. Yakovlev, Phys. Rev. D 62 (2000) 114002 arXiv:hep-ph/0001297.

[56] P. Ball and R. Zwicky, Phys. Rev. D 71 (2005) 014015 arXiv:hep-ph/0406232.

[57] B. Melic, Phys. Lett. B 591 (2004) 91 arXiv:hep-ph/0404003.

[58] Y. M. Wang and C. D. Lü, Phys. Rev. D 77 (2008) 054003 arXiv:0707.4439 [hep-ph]].

[59] V. M. Braun, A. Lenz, N. Mahnke and E. Stein, Phys. Rev. D 65 (2002) 074011 arXiv:hep-ph/0112085.

[60] A. Lenz, M. Wittmann and E. Stein, Phys. Lett. B 581 (2004) 199 arXiv:hep-ph/0311082.

[61] V. M. Braun, A. Lenz and M. Wittmann, Phys. Rev. D 73 (2006) 094019 arXiv:hep-ph/0604050.

[62] V. M. Braun, D. Y. Ivanov, A. Lenz and A. Peters, Phys. Rev. D 75 (2007) 014021 arXiv:hep-ph/0611386.

[63] M. Q. Huang and D. W. Wang, Phys. Rev. D 69 (2004) 094003 arXiv:hep-ph/0401094.

[64] M. Q. Huang and D. W. Wang, arXiv:hep-ph/0608170.

[65] V. M. Braun, A. Lenz, G. Peters and A. V. Radyushkin, Phys. Rev. D 73 (2006) 034020 arXiv:hep-ph/0510237.

[66] Z. G. Wang, J. Phys. G 34 (2007) 493 arXiv:hep-ph/0609155.

[67] T. M. Aliev and A. Ozpineci, Nucl. Phys. B 732 (2006) 291 arXiv:hep-ph/0406331.

[68] T. M. Aliev, A. Ozpineci, S. B. Yakovlev and V. Zamiralov, Phys. Rev. D 74 (2006) 116001. 
[69] P. Ball, V. M. Braun, Y. Koike and K. Tanaka, Nucl. Phys. B 529 (1998) 323 arXiv:hep-ph/9802299.

[70] P. Ball and V. M. Braun, Nucl. Phys. B 543 (1999) 201 arXiv:hep-ph/9810475.

[71] P. Ball and M. Boglione, Phys. Rev. D 68 (2003) 094006 arXiv:hep-ph/0307337.

[72] V. M. Braun and A. Lenz, Phys. Rev. D 70 (2004) 074020 arXiv:hep-ph/0407282.

[73] P. Ball and R. Zwicky, Phys. Lett. B 633 (2006) 289 arXiv:hep-ph/0510338.

[74] P. Ball, V. M. Braun and A. Lenz, JHEP 0605 (2006) 004 arXiv:hep-ph/0603063.

[75] P. Ball, V. M. Braun and A. Lenz, JHEP 0708 (2007) 090 arXiv:0707.1201 [hep-ph]].

[76] V. Braun, R. J. Fries, N. Mahnke and E. Stein, Nucl. Phys. B 589 (2000) 381 [Erratum-ibid. B 607 (2001) 433] arXiv:hep-ph/0007279.

[77] X. d. Ji, J. P. Ma and F. Yuan, Nucl. Phys. B 652 (2003) 383 arXiv:hep-ph/0210430.

[78] X. d. Ji, J. P. Ma and F. Yuan, Phys. Rev. Lett. 90 (2003) 241601 arXiv:hep-ph/0301141.

[79] X. d. Ji, J. P. Ma and F. Yuan, Eur. Phys. J. C 33 (2004) 75 arXiv:hep-ph/0304107.

[80] V. M. Braun, Talk given at 7th Workshop on Continuous Advances in QCD, Minneapolis, Minnesota, 11-14 May 2006, arXiv:hep-ph/0608231.

[81] F. Legger and T. Schietinger, Phys. Lett. B 645 (2007) 204 [Erratum-ibid. B 647 (2007) 527] arXiv:hep-ph/0605245.

[82] G. Hiller, M. Knecht, F. Legger and T. Schietinger, Phys. Lett. B 649 (2007) 152 arXiv:hep-ph/0702191.

[83] C. K. Chua, X. G. He and W. S. Hou, Phys. Rev. D 60 (1999) 014003 arXiv:hep-ph/9808431.

[84] G. Buchalla, A. J. Buras and M. E. Lautenbacher, Rev. Mod. Phys. 68 (1996) 1125 arXiv:hep-ph/9512380.

[85] C.S. Kim, T. Morozumi, A.I. Sanda, Phys. Lett. B 218 (1989) 343.

[86] X. G. He, T. D. Nguyen and R. R. Volkas, Phys. Rev. D 38 (1988) 814.

[87] B. Grinstein, M.J. Savage, M.B. Wise, Nucl. Phys. B 319 (1989) 271.

[88] N. G. Deshpande, J. Trampetic and K. Panose, Phys. Rev. D 39 (1989) 1461.

[89] P. J. O'Donnell and H. K. K. Tung, Phys. Rev. D 43 (1991) 2067.

[90] N. Paver and Riazuddin, Phys. Rev. D 45 (1992) 978.

[91] A. Ali, T. Mannel and T. Morozumi, Phys. Lett. B273 (1991) 505.

[92] D. Melikhov, N. Nikitin and S. Simula, Phys. Lett. B 430 (1998) 332 arXiv:hep-ph/9803343].

[93] J. M. Soares, Nucl. Phys. B 367 (1991) 575.

[94] G. M. Asatrian and A. Ioannisian, Phys. Rev. D 54 (1996) 5642 arXiv:hep-ph/9603318.

[95] M. R. Ahmady, Phys. Rev. D 53 (1996) 2843 arXiv:hep-ph/9508213.

[96] F. Kruger and L. M. Sehgal, Phys. Lett. B 380 (1996) 199 arXiv:hep-ph/9603237.

[97] Z. Ligeti, I. W. Stewart and M. B. Wise, Phys. Lett. B 420 (1998) 359 arXiv:hep-ph/9711248.

[98] N. G. Deshpande, X. G. He and J. Trampetic, Phys. Lett. B 367 (1996) 362.

[99] J. M. Soares, Phys. Rev. D 53 (1996) 241 arXiv:hep-ph/9503285.

[100] G. Hiller and A. Kagan, Phys. Rev. D 65 (2002) 074038 arXiv:hep-ph/0108074. 
[101] V. M. Braun, G. P. Korchemsky and D. Mueller, Prog. Part. Nucl. Phys. 51 (2003) 311 arXiv:hep-ph/0306057.

[102] A.P. Bakulev and A.V. Radyushkin, Phys. Lett. B 271 (1991) 223.

[103] S.V. Mikhailov and A.V. Radyushkin, Phys. Rev. D 45 (1992) 1754.

[104] B. L. Ioffe, Nucl. Phys. B188, 317 (1981) [Erratum-ibid. B 191 (1981) 591].

[105] V. M. Belyaev and B. Y. Blok, Z. Phys. C30 (1986) 151.

[106] M. A. Ivanov, J. G. Korner, V. E. Lyubovitskij, M. A. Pisarev and A. G. Rusetsky, Phys. Rev. D 61 (2000) 114010 arXiv:hep-ph/9911425.

[107] Y. B. Dai, C. S. Huang, C. Liu and C. D. Lu, Phys. Lett. B 371 (1996) 99 arXiv:hep-ph/9602242.

[108] A. G. Grozin and O. I. Yakovlev, Phys. Lett. B 285 (1992) 254.

[109] D. W. Wang and M. Q. Huang, Phys. Rev. D 67 (2003) 074025 arXiv:hep-ph/0302193.

[110] I.I. Balitsky and V.M. Braun, Nucl. Phys. B 311 (1989) 541.

[111] A. Khodjamirian and R. Ruckl, Adv. Ser. Direct. High Energy Phys. 15 (1998) 345 arXiv:hep-ph/9801443.

[112] M. Diehl, T. Feldmann, R. Jakob and P. Kroll, Eur. Phys. J. C 8 (1999) 409 arXiv:hep-ph/9811253.

[113] V. M. Braun and I. E. Halperin, Phys. Lett. B 328 (1994) 457 arXiv:hep-ph/9402270.

[114] V. M. Braun, A. Khodjamirian and M. Maul, Phys. Rev. D 61 (2000) 073004 arXiv:hep-ph/9907495.

[115] W.M. Yao et al., J. Phys. G 33, 1 (2006).

[116] B.L. Ioffe, Prog. Part. Nucl. Phys. 56, 232 (2006) [arXiv: hep-ph/0502148.

[117] C. W. Bauer, Z. Ligeti, M. Luke, A. V. Manohar and M. Trott, Phys. Rev. D 70 (2004) 094017 arXiv:hep-ph/0408002.

[118] V. L. Chernyak, A. A. Ogloblin and I. R. Zhitnitsky, Z. Phys. C 42, 569 (1989).

[119] G. R. Farrar, H. Zhang, A. A. Ogloblin and I. R. Zhitnitsky, Nucl. Phys. B 311 (1989) 585.

[120] H. n. Li and B. Tseng, Phys. Rev. D 57 (1998) 443 arXiv:hep-ph/9706441.

[121] M. Neubert, Invited plenary talk delivered at the 23rd International Symposium on Lepton and Photon Interactions at High Energy (Daegu, Korea, 13-18 August 2007) arXiv:0801.0675 [hep-ph]. 